\title{
Characterization of Land Suitability for Crop and Fruit Production in Wadi Sakher at North West Coastal Zone of Egypt
}

\author{
Abdalsamad Abdalsatar Ali Aldabaa
}

\begin{abstract}
Land suitability measures the land performance for cultivated crops based on soil qualities. The current study is to evaluate the land suitability of an area under rainfed agriculture system that is depended mainly on harvesting of seasonal rainfall. An integrated approach combined Land Use Suitability Evaluation Tool (LUSET) with the local farmer expertise and knowledge was used. Based on the farmer expertise and knowledge, three classes landform priorities were proposed for the selected crops; watermelon, barley, wheat, olive, fig, pomegranate, and almond. These classes were named as high (P1), moderate (P2), and low priority (P3). The study area was delineated into four main landforms called, coastal plain, piedmont plain, escarpment, and tableland. Piedmont plain was reclassified into subunits named as coalesced delta, drainage channel, and summit, while escarpment and tableland reclassified into two subunit; drainage channel and summit. Eight soil mapping units are classified according to variation in slope, soil depth, and soil texture. Based on physical, chemical, and fertility status of these soil mapping units, they differ in their soil suitability response for the selected crops. the study found that, SMU01 and 02 are marginal suitable, while SMU03 and 04 differ from high suitable to moderate suitable for the selected crops. SMU05 and 08 are moderate suitable for watermelon, barley, and wheat while they are marginal suitable for orchard trees. Soil mapping units 06 and 07 are not suitable for orchard tress and marginal suitable for watermelon, barley, and wheat. As for the landform priority, the study revealed that the coastal plain has low priority for orchard trees and watermelon, while it has high and moderate priority for barley and wheat, respectively. The coalesced deltas has high priority for barley and wheat while it has moderate priority for watermelon and orchard trees. The drainage channel is highly priority for watermelon and orchard trees while it is low priority for barley and wheat. On the other hand, summit has high priority for barley and wheat, moderate for watermelon, low for orchard trees.
\end{abstract}

Key words; Suitability

Rainfed

watermelon barley wheat orchard

\section{INTRODUCTION}

Because of the human population and the increasing of their activity, land becomes a rare resource and keeps to be under stress by competing landuse patterns. Resolving the disagreeing demands of different varieties of landuses for land and choosing the best land use asks for a decision making process that is based on a clear understanding of the chances and limits offered by the relatively permanent land valuable supplies. Recently, the population over the world is increasing dramatically, (Liu and Chen, 2006). Lal (1994) reported that the land capability for crop production to fulfill the demand of the doubled increasing population is decreasing as the cause of the sever soil degradation. Moreover, the severe declination of land's productivity ability has taken place on over $10 \%$ of the earth's cultivated areas as a result of soil erosion, excessive tillage, overgrazing, etc.

The land suitability defines the performance of a given area of land for a specific kind of land use, moreover, it is usually determined on the suppose that the defines land use will be continued and the environmental quality must be protected of even amended on the site and the surrounding area . Moreover, the suitability is consider as a function of crop requirements and land properties and it measures how the soil quality will matches the particular landuse requirements. The define of crop land suitability is a prerequisite to attain optimum utilization of the obtainable land resources for agricultural production in the way of sustainability,(FAO, 1976). Sathish and Niranjana (2010) reported that production could be attained through well-thought-out survey of the soils, figuring out the worth, amount, or quality of their potentials for wide range of land use options and creating land use plans which were economically doable, socially acceptable and helpful to the earth.

Remote sensing (RS) data are used for guessing a number of biophysical limits and indices cropping systems analysis, in addition to monitor the changes occur in both land use and land cover during different seasons, (Rao et al., 1996). AbdelRahman et al. (2016) quoted that RS data can be used to describe different physiographic units besides getting helping thing information about site traits, like slope, direction and aspect of a given area, however, explained information of soil worth. Because of this, soil survey data are extermely important to define a soil mapping units of a given area, which helps in getting crop suitability and cropping system analysis. Remote sensing data can be combined with soil survey information through

\footnotetext{
${ }^{1}$ Desert Research Center

Corresponding author: (abdelstar2004@gmail.com)

Received OCTOBR 11, 2018, Accepted November 11, 2018
} 
geographical information system (GIS) to evaluate crop suitability under different biophysical conditions. Beek et al. (1997) and Merolla et al. (1994) applied that modeling of GIS coupling with remotely sensed data for quantitative land evaluation. Land cover patterns reflect the natural and social processes, in which important information for modeling are provided to understand many important events or patterns on the earth, (Liang, 2008). To create thematic maps that is representing land use land cover (LULC) using classification of the available satellite images is one of the valuable common application of remote sensing, (Yan et al., 2015). Moreover, Remote sensing has long been important and effective for supervising land cover with its ability to quickly provide broad, exact, fair and unprejudiced available information related to the existing space of changing over time at different places of the land surface (Hansen et al., 2000). There are different tools for mapping and distinguishing land use land cover (LULC) such as unsupervised classification, supervised classification, and normalized difference vegetation index(NDVI), (Samaniego and Schulz, 2009) and (Aredehey et al., 2018). Yadav et al. (2012) demonstrated that LULC maps supply reliable land use information of the land environment.

Rainfed agriculture is defined as a type of cultivated land use that depends on seasonal rainfall, (Doolette, 1986). Heathcote (1983) reported that rainfed system is characterized by the processing of soil to enable the growing plants to sustain maximum moisture. On contrary, under the North Western Coast of Egypt, rainfed agriculture means by all processes that enable the cultivated plants to bear the minimum moisture especially during summer season. These could be attained by some of means or manipulations that be indirect through maximizing the soil moisture by constructing obstacle dykes (any known type), by selecting plants that can tolerate the expected moisture conditions, and by tillage practices to conserve soil moisture before and after planting or reduce loss of moisture after planting by evaporation.

The current study represents wadi sakher watershed at the north western coast of Egypt which is characterized by rainfed agricultural system. In order to perform the soil suitability in the studied area, land use and land cover (LULC) is one of the most prerequisite processes that has to be carried out. In the current study, land use land cover (LULC) is mapped using two of well-know techniques called the supervised classification and normalized difference vegetation index. The performance of the applied tools was evaluated under the study area circumstances. This study aimed at evaluating soils of wadi sakher to define degrees of crop suitability under rainfed conditions

\section{MATERIALS AND METHODS}

\section{Study area description}

Wadi sakher catchment is one of 218 watershed covering the area extended from Fuka to El Salloum at North Western Coast of Egypt. It is located between longitudes 551549.5 to and $554735.7 \mathrm{~m} \mathrm{E}$, and latitude 3443126.2 and $3451232.4 \mathrm{~m} \mathrm{~N}$ covering an area of about 3266 faddan, Figure (1A and B). According to the climatic data derived from https://globalweather.tamu.edu, it is found that the annual rainfall over the current study area decreases from $112 \mathrm{~mm}$ at the coastal shoreline to $98 \mathrm{~mm}$ at the extreme south border of the study area, Figure (1C).

Whatever, the study area is characterized by a long hot dry summer and short cool rainy winter. The monthly temperature is ranged from 14.4 to $26.8{ }^{\circ} \mathrm{C}$, wind speed averaged at $18.9 \mathrm{~km} / \mathrm{hr}$, and the relative humidity varies from 55 to $65 \%$. Based on the outlined by Soil Survey Staff (2014), soils are characterized by torric moisture and hyperthermic temperature regimes. As for the lithology of the study area, it is dominated by a sedimentary rocks varying from Tertiary period (middle Miocene) to Quaternary. The sediments of Middle Miocene are widely distributed in the tableland while the Quaternary deposits are differentiated from Pleistocene and Holocene formation that have a wide distribution and forms the bulk of the coastal plain, (El Shazly et al., 1975). From geomorphological point of view, El Shazly et al. (1975) demonstrated that the study area is characterized by the presence of the coastal plain then the piedmont plain and finally Libyan plateau formed by Miocene rocks with two distinct layers: an upper limestone component of middle Miocene age and lower fossiliferous limestone and marls of lower Miocene age.

The area is divided into three distinct micro AgroEcological Zones based on the agricultural activities. Zone I extends from the coastal plain to the plateau escarpment in which soils are characterized by deep depths. The agricultural production are predominated by fruit trees such as fig and olive interpolated by some cereal. Zone II extends from the plateau escarpment southward with $20 \mathrm{~km}$ in which cereals are dominated with fewer trees and. Zone III goes beyond the end of zone II and characterized by scattered cereals production. Drainage channels and their alluvial fans which are dissecting the limestone plateau are the main productive unit of orchard trees i.e. fig, olive, almond and pomegranates. 


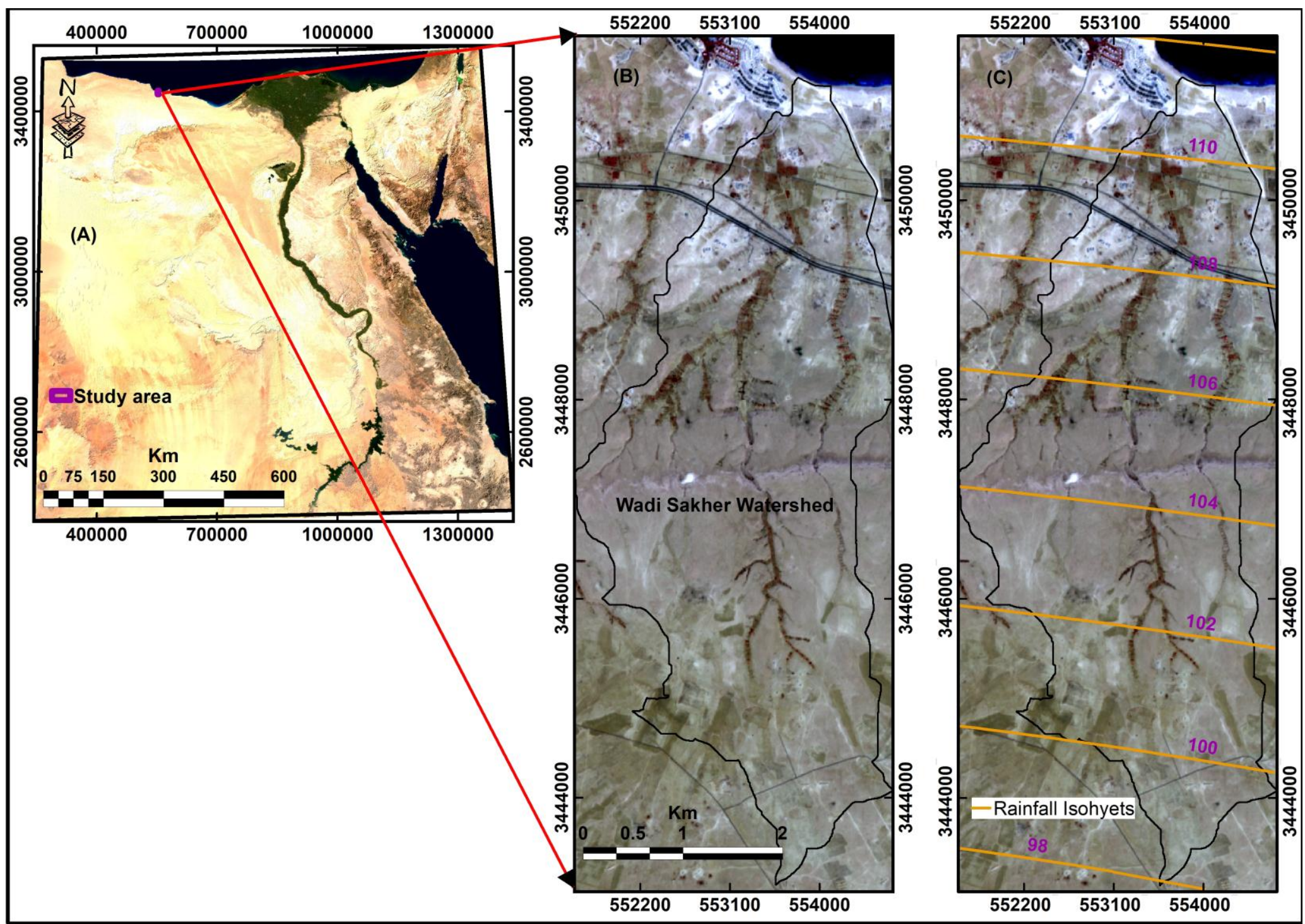

Figure 1. Location map of the study area (A): Egypt and the study area, (B): boundary of the study area, (C), rainfall isohyets covering the study area 


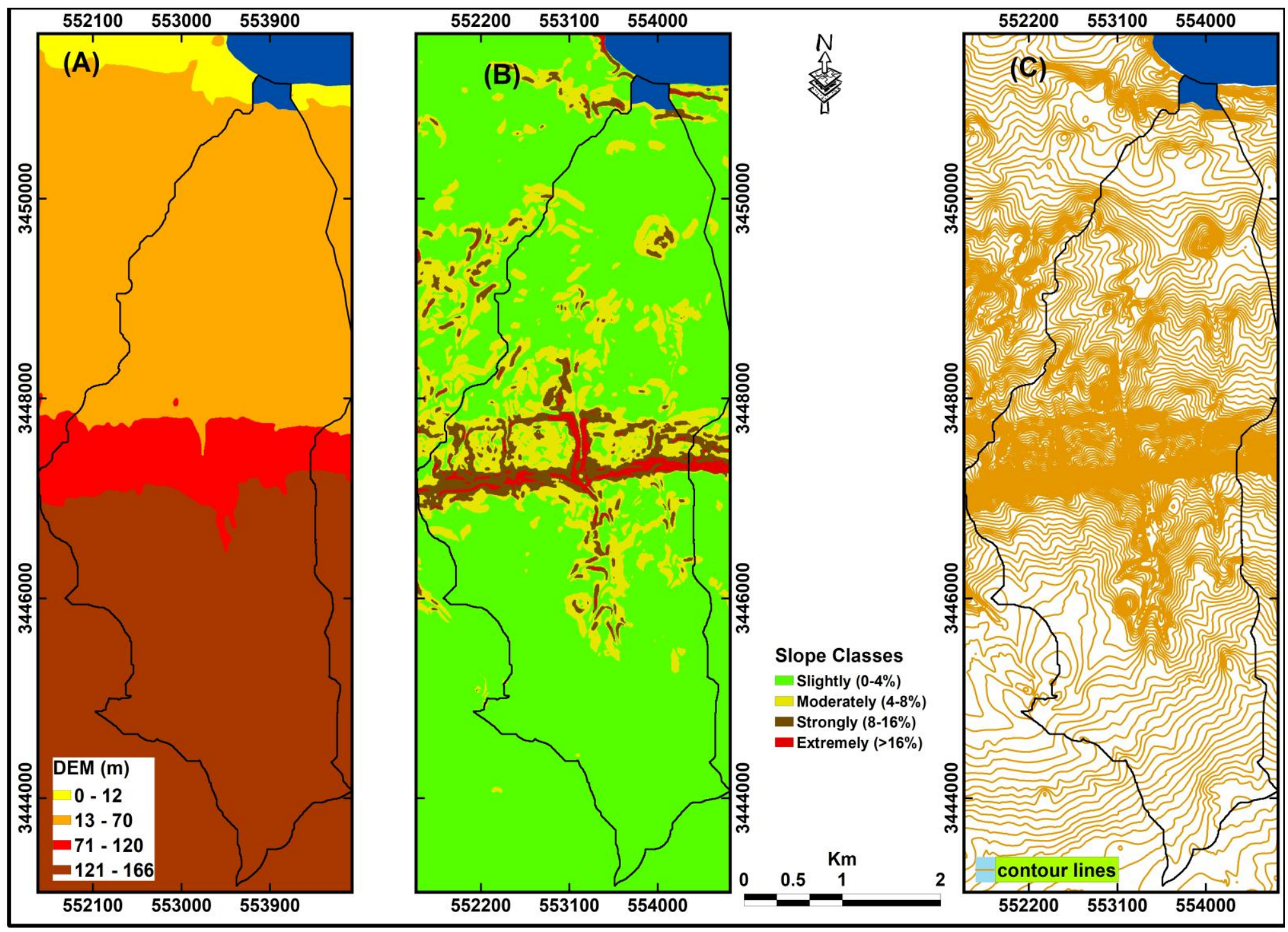

Figure 2. (A) digital elevation model, (B) Surface slope, and (C) contour lines of the studied area 


\section{Digital data collection and preprocessing}

Digital elevation model, (SRTM 30m) used for delineating the study area of wadi sakher watershed was downloaded from https://earthexplorer.usgs.gov, accordingly topographical parameters like degree and length of slope were extracted.. The downloaded DEM was enhanced by digitizing the contour lines and elevation spots of the 1: 25,000 topographic maps, sheet No. NH35 02a1 and NH35 02a3. The watershed delineation was performed using ArcSWAT model. After delineating wadi Sakher watershed, the digital elevation model (DEM) was clipped to the extent of the studied area and parts of its neighboring areas, Figure (2A, B, and C). The available sentinel 2 satellite image (level-1c S2) for the study area covered by one scene was downloaded from the European Space Agency's (ESA) sentinel scientific Data Hub, Figure (1B). The current study selected temporal image with less than 10 $\%$ cloud coverage, taken in August 28, 2017. The visible bands 2, 3 and 4 and the near-infrared band (band8) of Sentinel-data were applied after band combination for running NDVI that was used for extracting the landuselandcover (LULC) by applying supervised classification. The supervised classification was achieved using ground checkpoints (160 points) which were demarcated and described through the field truth using Global Positioning System (GPS Garmin 680). Furthermore, LULC was classified by running the maximum likelihood classifier which quantitatively evaluates both the variance and covariance of the category spectral response patterns when classifying an unknown pixel so it is one of the most accurate classifier. The resulted maps of NDVI and LULC were also clipped to the extent of the studied area. By using the Sentinel digital image and the enhanced digital elevation model, the landforms covering the study area was performed.

\section{Field works and laboratory analyses}

During implementing a project called " the Sustainable Agricultural Development at North Western Coast of Egypt (El-Kasaba / Paghoush basin) and funded by the Arab Center for the Studies of Arid Zones and Dry Lands (ACSAD) from 2014 to 2017), Soils of the studied area were surveyed using 68 soil profiles representing the dominant landforms covering the studied area, 27 out of them were described as rock exposures. These soil profiles were described according to (Jahn et al., 2006) was processed. The soil horizons were sampled for laboratory analyses included the measurement of gravel volume, soil texture, soil water characteristics (Field capacity, wilting point, and available water were calculated), electrical conductivity, $\mathrm{pH}$, calcium carbonate, sodium absorption ratio, organic matter, and cation exchangeable capacity were measured according to (USDA, 2004). Sodium exchangeable percent is mathematically calculated according to (Rashidi and Seilsepour, 2008).

\section{Land evaluation classification}

Land suitability evaluation was undertaken on the base of (FAO, 1976) and (FAO, 2007). In the current study a land suitability evaluation program called Land Use Suitability Evaluation Tool (LUSET) programmed by (Yen et al., 2006). LUSET is programmed in Microsoft Excel and is simple to use. The processes and calculations in LUSET were coded using Visual Basic for Application (VBA). The crop requirements are categorized into four groupings of factors: Terrain, Soil characteristics (physical and chemical soil properties), Temperature and Water. The requirements for most of the commonly grown crops used in this program are provided by (Sys et al., 1993). Whereas the climatic conditions are not varied through the extent of the studied area so it was canceled as well as the water properties hence the main source of the irrigation water in the studied area is rainfall that is harvested in the stream bed by using different techniques of dykes.

Accordingly, the current study was dependent on the terrain (surface slope) and soil characteristics to evaluate the studied area for watermelon, olive, fig, wheat, barley, pomegranate, and almond. Under the circumstance of the study area and based on the farmer expertise and knowledge, landforms and or land elements play a great role in chosen types of the cultivated crops. So the landform priority for the selected crops was investigated in the current study and classified into three categories; high priority (P1), moderate priority (P2), and less priority (P3). This proposed priority was classified in relation to the soil quality, the quality of practices for managed, and/or the quality of water harvesting.

\section{RERSULTS AND DISCUSSION}

\section{Physiographic units}

Based on the high resolution digital image (Sentinel $2 \mathrm{~A}$, with $10 \mathrm{~m}$ resolution) and the modified digital elevation model (DEM with $5 \mathrm{~m}$ resolution), four landforms were achieved over the study area .Each landform was represented by some soil profiles, (Figure $3)$.

\section{The coastal plain}

The coastal plain is parallel to the present Mediterranean shoreline and covers an area of about 24 faddan and was represented by 2 soil profiles. This unit is occupied by yellowish brown sediments with thickness greater than $100 \mathrm{~cm}$. this formation may be existed by intensive water erosion due to presence of intermittent streams which drain from the tableland area. 
These area is covered by typical hummocky surface and characterized by quite coarse-texture soils. The soil of this unit as shown in Table (1) is generally deep, the vertical soil layers differ from coarse-texture to moderately fine texture with no gravel. The soils are extremely saline where values of electrical conductivity are more than $16 \mathrm{dS} / \mathrm{m}$ in most of layers. The soils react as moderately alkaline as categorized by Soil Science Division Staff ( 2017), where the soil $\mathrm{pH}$ ranges from 7.9 to 8.4 . Soil calcium carbonate ranges from strongly to extermely calcareous as categorized by (Jahn et al., 2006), where its value ranges from 18.2 to $64.81 \%$. Hence, soils of coastal plain could be classified as saline-sodic soils, where soils salinity $>4 \mathrm{dS} / \mathrm{m}, \mathrm{pH}<$ 8.5, and SAR > 13, (Kamphorst and Bolt, 1976). However, the soil of the coastal plain has a very low value of organic matter not more than $1 \%$ due to the soil temperature regime. This unit has in general low to moderate cation exchangeable capacity (ranges from 4.29 to $12.32 \mathrm{cmol} / \mathrm{kg}$ soil) due to the soil texture and organic matter.

\section{Piedmont plain}

The piedmont plain covers an area of about 1445.6 faddan and was represented by 23 soil profiles, 9 out of them were described as rock exposures. It is well developed where the tableland escarpment are well defined. Its surface is characterized by elongated ridges and low depression and either covered with thin layer of alluvial and sand deposits or degraded and appears as rocky surface. By using the modified DEM and highly resolution satellite image, this unit were subdivided into three subunits of landforms, namely coalesced delta, drainage channel, and summit, and they briefly discuss as following.

\section{Piedmont coalesced Delta}

A continuous surface of coalesced alluvial delta, formed along the base of the piedmont plain before the appearing of coastal plain, presents a gentle slope. This subunit, covering an area of about 269.6 faddan, was represented by 4 soil profiles that is described as deep soil profiles (depth $>100 \mathrm{~cm}$ ). The distribution of gravel content varies from none to common $(0-11.50 \%)$ but it does not affect the modification of soil texture as stated by (Schoeneberger, 2012). The soil texture is coarsetexture to moderately coarse-texture soil, accordingly the depth of available water is very low (less than $10 \%$ ). The soils of this subunit are none saline where the electrical conductivity is less than $2 \mathrm{dS} / \mathrm{m}$, except the soils of profile 6 , the soil salinity is very slightly saline (EC ranges from 2.69 to $4.32 \mathrm{dS} / \mathrm{m}$ ). The soil reaction measured by $\mathrm{pH}$ showed that the soils of this subunit react as moderately alkaline. The soils are defined as extermely calcareous soils where calcium carbonate is greater than $25 \%$ overall the profile layers. Taking SAR (less than 13), EC, and $\mathrm{pH}$ in consideration, this subunit could be described as fresh soils. The soil fertility status measured by soil organic carbon and cation exchangeable capacity is very low due to the low value of both them.

\section{Piedmont drainage channels}

The stream channel of the wadi system are recognized in the piedmont plain. This subunit covers an area of about 388 faddan and was represented by 7 soil profiles. These stream pour their water and sediments in the coalesced fans. The soil depth varies from moderately deep to deep and they are filled with calcareous (calcium carbonate ranges from 17.2 to 60 $\%)$ coarse to moderately coarse-texture soils. The gravel content generally is less than $15 \%$ except the subsurface layer of soil profile 7 and profile 13 where the soil texture of these layer was modified to very gravelly coarse-texture soils. As well, the soil texture of the surface layer of soil profile 9 was modified into gravelly coarse-texture soil. As mentioned before, the soil water characteristics especially the available water affected by the soil texture are very low where the available water not exceeds $10 \%$. Some of soils of this subunit are defined as fresh soils and other as saline soils interpreted from the data of EC, $\mathrm{pH}$, and SAR. The soil fertility of this subunit is also poor as indicated by the values of organic matter and cation exchangeable capacity.

\section{Piedmont summit}

This subunit covers an area of about 788.4 faddan and was represented by 12 soil profiles 9 out of them described as rock exposures. Accordingly, majority of this area is rocky area with very few exceptional spots represented by soil profiles 14,15 , and 16 . The soil depth of these spots ranges from very shallow to moderately deep soils. The gravel content ranges from 2.7 to $22.2 \%$. The soil texture in general is coarsetexture except the subsurface layer of soil profile 15 is moderately coarse-texture soil. Taking the percentage of soil gravel in consideration, the soil texture of the surface layer of soil profile 15 , and 16 , respectively, could be modified into gravelly coarse-texture soils. Based on the values of Soil EC, $\mathrm{pH}$, and SAR, two types of soils were defined that are fresh and saline soils. The soil of this unit is calcareous where calcium carbonate content ranges from 26.5 to $60.3 \%$. The soil fertility distinguished by $\mathrm{OM}$ and $\mathrm{CEC}$ values is poor.

\section{Plateau Escarpment}

It is a steep slope or long cliff and it dissected by very deep and narrow stream channels. This area covers an area of about 450.2 faddan. It is subdivided into two 


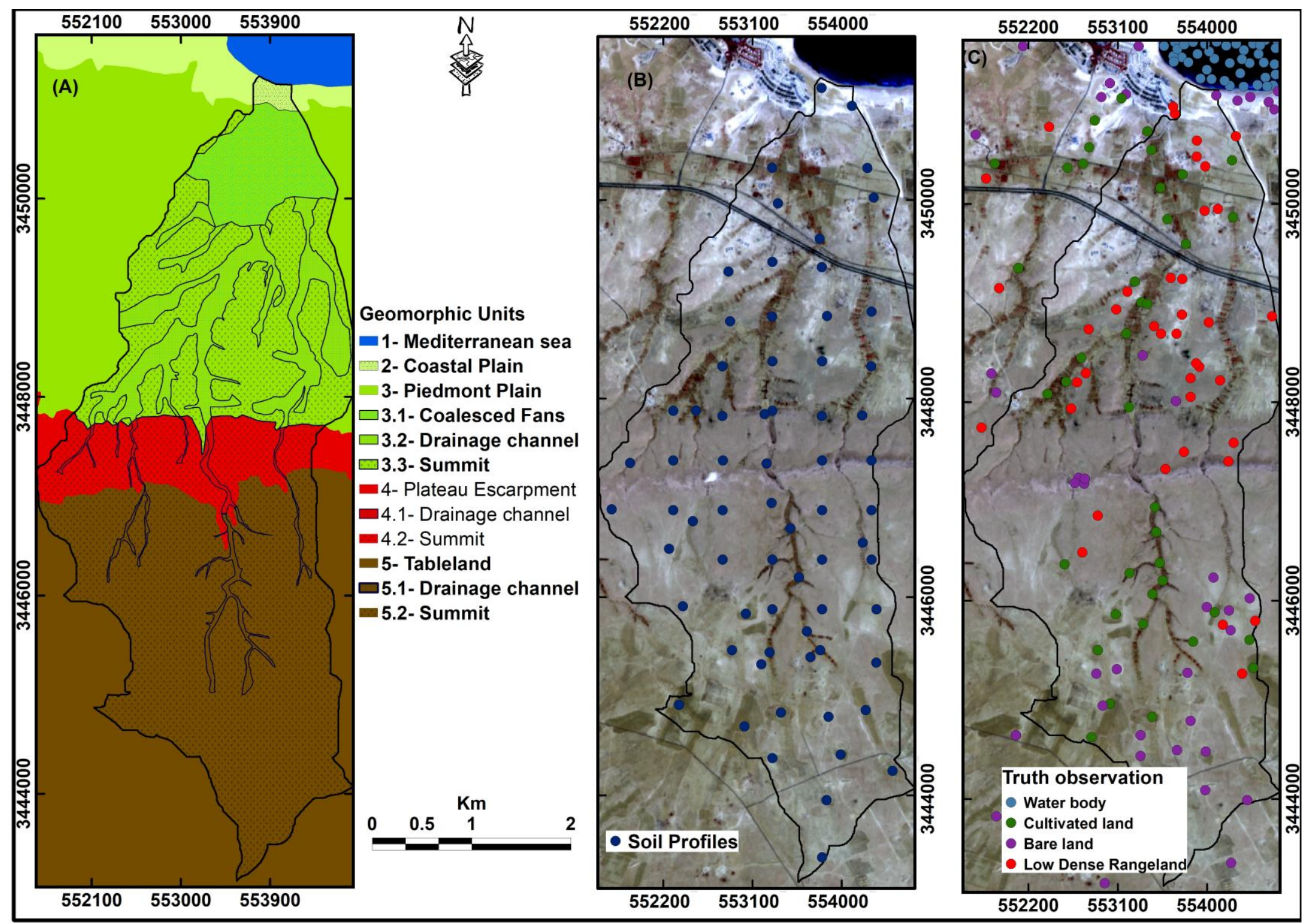

Figure 3.landforms covering the study area (A), soil profiles representing landforms (B), and Truth observation for landuse/landcover (C) 
Table 1. Some physical and chemical properties of the studied profiles, Wadi sakher

\begin{tabular}{|c|c|c|c|c|c|c|c|c|c|c|c|c|c|c|c|c|}
\hline \multirow[t]{2}{*}{$\begin{array}{c}\text { Soil } \\
\text { Profile }\end{array}$} & \multirow[t]{2}{*}{$\begin{array}{l}\text { Depth } \\
\text { (cm) }\end{array}$} & \multirow{2}{*}{$\begin{array}{c}\text { Gravel } \\
\mathbf{S} \\
\%\end{array}$} & \multicolumn{3}{|c|}{$\begin{array}{c}\text { Particles size } \\
\text { distribution }\end{array}$} & \multirow[t]{2}{*}{ Texture } & \multirow[t]{2}{*}{$\begin{array}{c}\text { F.C } \\
\%\end{array}$} & \multirow[t]{2}{*}{$\begin{array}{c}\text { W.P } \\
\%\end{array}$} & \multirow[t]{2}{*}{$\begin{array}{c}\text { A.W } \\
\%\end{array}$} & \multirow[t]{2}{*}{$\begin{array}{c}\text { S.P } \\
\%\end{array}$} & \multirow[t]{2}{*}{$\begin{array}{c}\text { EC } \\
\mathrm{dS} / \mathrm{m}\end{array}$} & \multirow[t]{2}{*}{ pH } & \multirow[t]{2}{*}{$\begin{array}{c}\mathrm{CaCO}_{3} \\
\%\end{array}$} & \multirow[t]{2}{*}{ SAR } & \multirow[t]{2}{*}{$\underset{\%}{\mathrm{OM}}$} & \multirow[t]{2}{*}{$\begin{array}{c}\text { CEC } \\
\mathrm{meq} / 100 \mathrm{~g}\end{array}$} \\
\hline & & & Sand & Silt & Clay & & & & & & & & & & & \\
\hline \multicolumn{17}{|c|}{ Coastal Plain (24 faddan) } \\
\hline \multirow[t]{5}{*}{1} & $0-15$ & 0.00 & 68.20 & 19.11 & 12.69 & Sandy loam & 19.77 & 9.96 & 9.81 & 42.33 & 62.5 & 8.40 & 23.19 & 36.08 & 0.77 & 9.27 \\
\hline & $15-40$ & 0.00 & 55.41 & 26.68 & 17.91 & Sandy loam & 23.18 & 11.98 & 11.20 & 45.17 & 27.5 & 7.90 & 28.88 & 19.84 & 0.67 & 11.50 \\
\hline & $40-75$ & 0.00 & 76.88 & 17.51 & 5.61 & Loamy sand & 20.42 & 12.29 & 8.13 & 43.65 & 13.19 & 8.00 & 50.93 & 13.20 & 0.51 & 4.74 \\
\hline & $75-120$ & 0.00 & 61.99 & 17.78 & 20.23 & Sandy loam & 22.93 & 13.06 & 9.88 & 45.37 & 9.79 & 8.10 & 64.81 & 11.62 & 0.58 & 12.32 \\
\hline & $120-150$ & 0.00 & 90.87 & 5.87 & 3.26 & Sand & 11.95 & 4.72 & 7.23 & 33.16 & 16.71 & 8.00 & 63.78 & 14.83 & 0.70 & 4.29 \\
\hline \multirow[t]{4}{*}{2} & 0-20 & 0.00 & $\mathbf{3 7 . 5 3}$ & 45.03 & 17.44 & Loam & 26.04 & 11.53 & 14.52 & 46.32 & 68.30 & 8.20 & 27.74 & 38.77 & 0.77 & 11.65 \\
\hline & $20-50$ & 0.00 & 46.75 & 45.84 & 7.41 & Loam & 20.83 & 8.22 & 12.61 & 40.18 & 36.20 & 8.10 & 24.31 & 23.87 & 0.67 & 6.25 \\
\hline & $50-85$ & 0.00 & 48.98 & 44.85 & 6.74 & Loam & 22.69 & 8.34 & 14.35 & 40.22 & 22.7 & 8.10 & 24.02 & 17.61 & 0.57 & 5.54 \\
\hline & $85-115$ & 0.00 & 63.19 & 18.40 & 18.41 & Sandy loam & 22.20 & 12.31 & 9.89 & 44.76 & 23.30 & 8.10 & 18.22 & 17.89 & 0.36 & 10.57 \\
\hline \multicolumn{17}{|c|}{ Piedmont plain, Coalesced Delta (269.6 faddan) } \\
\hline 3 & $0-24$ & 1.30 & 92.00 & 2.50 & 5.50 & Sand & 13.10 & 5.95 & 7.14 & 35.98 & 0.65 & 7.88 & 78.7 & 7.38 & 0.92 & 4.83 \\
\hline & $24-60$ & 0.00 & 97.00 & 2.50 & 0.50 & Sand & 8.55 & 2.70 & 5.85 & 22.33 & 0.28 & 7.96 & 83.9 & 7.21 & 0.60 & 0.93 \\
\hline & $60-100$ & 0.00 & 97.00 & 2.50 & 0.50 & Sand & 8.55 & 2.70 & 5.85 & 22.33 & 0.29 & 8.33 & 90.3 & 7.21 & 0.43 & 0.45 \\
\hline 4 & $0-20$ & 0.00 & 84.50 & 10.00 & 5.50 & Loamy sand & 14.43 & 6.28 & 8.15 & 36.52 & 0.54 & 8.07 & 35.6 & 7.33 & 1.08 & 5.27 \\
\hline & $20-55$ & 0.00 & 84.50 & 10.00 & 5.50 & Loamy sand & 14.43 & 6.28 & 8.15 & 36.52 & 0.54 & 8.07 & 35.6 & 7.33 & 0.89 & 4.74 \\
\hline & $55-90$ & 0.00 & 84.50 & 5.00 & 10.50 & Loamy sand & 16.67 & 8.83 & 7.83 & 40.10 & 0.46 & 8.28 & 40.4 & 7.29 & 0.55 & 6.79 \\
\hline & $90-110$ & 0.00 & 87.00 & 7.50 & 5.50 & Loamy sand & 13.97 & 6.17 & 7.80 & 36.34 & 0.56 & 8.38 & 46.6 & 7.33 & 0.43 & 3.45 \\
\hline & $110-130+$ & 0.00 & 82.00 & 10.00 & 8.00 & Loamy sand & 15.98 & 7.64 & 8.34 & 38.78 & 0.44 & 8.44 & 42.0 & 7.28 & 0.43 & 4.95 \\
\hline 5 & 0-30 & 11.50 & 84.50 & $\mathbf{1 0 . 0 0}$ & 5.50 & Loamy sand & 14.43 & 6.28 & 8.15 & 36.52 & 0.54 & 8.07 & 35.6 & 7.33 & 1.08 & 5.27 \\
\hline & $30-60$ & 0.00 & 84.50 & 10.00 & 5.50 & Loamy sand & 14.43 & 6.28 & 8.15 & 36.52 & 0.54 & 8.07 & 35.6 & 7.33 & 0.89 & 4.74 \\
\hline & $60-90$ & 4.50 & 84.50 & 5.00 & 10.50 & Loamy sand & 16.67 & 8.83 & 7.83 & 40.10 & 0.46 & 8.28 & 40.4 & 7.29 & 0.55 & 6.79 \\
\hline & $90-120$ & 6.30 & 87.00 & 7.50 & 5.50 & Loamy sand & 13.97 & 6.17 & 7.80 & 36.34 & 0.55 & 8.38 & 46.6 & 7.33 & 0.43 & 3.45 \\
\hline & $120-150$ & 4.20 & 82.00 & 10.00 & 8.00 & Loamy sand & 15.98 & 7.64 & 8.34 & 38.78 & 0.44 & 8.44 & 42.0 & 7.28 & 0.43 & 4.95 \\
\hline 6 & 0-20 & 2.90 & 89.50 & 7.50 & 3.00 & Sand & 12.07 & 4.66 & 7.42 & 32.80 & 2.69 & 8.16 & 62.3 & 8.33 & 0.43 & 1.95 \\
\hline & $20-60$ & 2.70 & 72.00 & 10.00 & 18.00 & Sandy loam & 19.87 & 12.40 & 7.47 & 43.27 & 4.18 & 8.20 & 55.3 & 9.02 & 0.43 & 10.95 \\
\hline & $60-100$ & 0.00 & 72.00 & 10.00 & 18.00 & Sandy loam & 19.87 & 12.40 & 7.47 & 43.27 & 3.22 & 8.34 & 40.8 & 8.57 & 0.43 & 10.95 \\
\hline & $100-130$ & 0.00 & 79.50 & 12.50 & 8.00 & Loamy sand & 20.12 & 12.37 & 7.75 & 43.45 & 4.32 & 8.37 & 27.5 & 9.08 & 0.43 & 4.95 \\
\hline & & & & & & Piedmont plain & Draina & e Chan & el (388 & Iddan) & & & & & & \\
\hline 7 & 0-27 & 4.40 & 89.50 & 7.50 & 3.00 & Sand & 12.07 & 4.66 & 7.42 & 32.80 & 0.68 & 8.30 & 49.3 & 9.08 & 0.82 & 3.05 \\
\hline & $27-55$ & 55.60 & 84.50 & 7.50 & 8.00 & Loamy sand & 15.58 & 7.57 & 8.01 & 38.60 & 2.00 & 8.38 & 60.7 & 7.39 & 0.60 & 5.43 \\
\hline 8 & 0-30 & 1.60 & 79.50 & 12.50 & 8.00 & Loamy sand & 20.12 & 12.37 & 7.75 & 43.45 & 0.93 & 8.70 & 27.3 & 8.01 & 0.48 & 5.09 \\
\hline & $30-60$ & 1.80 & 82.00 & 10.00 & 8.00 & Loamy sand & 15.98 & 7.64 & 8.34 & 38.78 & 9.48 & 7.78 & 17.6 & 7.51 & 0.25 & 4.45 \\
\hline & $60-90$ & 3.00 & 84.50 & 10.00 & 5.50 & Loamy sand & 14.43 & 6.28 & 8.15 & 36.52 & 10.22 & 7.80 & 19.9 & 11.48 & 0.19 & 2.78 \\
\hline & $90-120$ & 0.00 & 84.50 & 10.00 & 5.50 & Loamy sand & 14.43 & 6.28 & 8.15 & 36.52 & 8.35 & 7.66 & 21.1 & 11.82 & 0.19 & 2.78 \\
\hline & $120-150$ & 0.00 & 87.00 & 10.00 & 3.00 & Sand & 12.62 & 4.82 & 7.80 & 32.98 & 6.52 & 7.80 & 17.2 & 10.95 & 0.21 & 1.34 \\
\hline 9 & 0-10 & 19.40 & 84.50 & 10.00 & 5.50 & Loamy sand & 14.43 & 6.28 & 8.15 & 36.52 & 5.48 & 8.05 & 36.4 & 10.10 & 0.77 & 4.41 \\
\hline & $10-45$ & 9.00 & 87.00 & 7.50 & 5.50 & Loamy sand & 13.97 & 6.17 & 7.80 & 36.34 & 8.12 & 8.16 & 44.7 & 9.62 & 0.77 & 4.41 \\
\hline & $45-70$ & 0.00 & 79.50 & 10.00 & 10.50 & Sandy loam & 17.36 & 8.90 & 8.46 & 40.47 & 6.73 & 8.17 & 38.1 & 10.84 & 0.26 & 5.98 \\
\hline 10 & $0-35$ & 2.30 & 89.50 & 7.50 & 3.00 & Sand & 12.07 & 4.66 & 7.42 & 32.80 & 3.5 & 8.3 & 29.2 & 10.20 & 0.81 & 3.02 \\
\hline & $35-75$ & 14.70 & 82.00 & 15.00 & 3.00 & Loamy sand & 13.77 & 5.14 & 8.63 & 33.34 & 5.7 & 8.02 & 25.1 & 8.70 & 0.81 & 3.02 \\
\hline
\end{tabular}


Continue.Table 1.

\begin{tabular}{|c|c|c|c|c|c|c|c|c|c|c|c|c|c|c|c|c|}
\hline \multirow[t]{2}{*}{ Soil Profile } & \multirow{2}{*}{$\begin{array}{c}\text { Depth } \\
\text { (cm) }\end{array}$} & \multirow{2}{*}{$\begin{array}{c}\text { Gravel s } \\
\% \\
\end{array}$} & \multicolumn{3}{|c|}{ Particles size distribution } & \multirow[t]{2}{*}{ Texture } & \multirow{2}{*}{$\begin{array}{c}\text { F.C } \\
\%\end{array}$} & \multirow{2}{*}{$\begin{array}{c}\text { W.P } \\
\%\end{array}$} & \multirow{2}{*}{$\begin{array}{c}\text { A.W } \\
\%\end{array}$} & \multirow{2}{*}{$\begin{array}{c}\text { S.P } \\
\%\end{array}$} & \multirow{2}{*}{$\begin{array}{c}E C \\
\mathrm{dS} / \mathrm{m}\end{array}$} & \multirow[t]{2}{*}{ pH } & \multirow{2}{*}{$\begin{array}{c}\mathrm{CaCO}_{3} \\
\% \\
\end{array}$} & \multirow[t]{2}{*}{ SAR } & \multirow{2}{*}{$\begin{array}{c}\text { OM } \\
\%\end{array}$} & \multirow{2}{*}{$\begin{array}{c}\text { CEC } \\
\mathrm{meq} / 100 \mathrm{~g}\end{array}$} \\
\hline & & & Sand & Silt & clay & & & & & & & & & & & \\
\hline \multirow[t]{5}{*}{11} & 0-30 & 11.50 & 84.50 & 10.00 & 5.50 & Loamy sand & 14.43 & 6.28 & 8.15 & 36.52 & 0.41 & 8.21 & 20.3 & 7.27 & 0.90 & 4.77 \\
\hline & $30-60$ & 0.00 & 79.50 & 12.50 & 8.00 & Loamy sand & 20.12 & 12.37 & 7.75 & 43.45 & 3.83 & 7.84 & 21.3 & 8.85 & 0.67 & 5.63 \\
\hline & $60-90$ & 4.50 & 79.50 & 10.00 & 10.50 & Sandy loam & 17.36 & 8.90 & 8.46 & 40.47 & 1.66 & 8.03 & 21.9 & 7.85 & 0.51 & 6.68 \\
\hline & $90-120$ & 6.30 & 84.50 & 10.00 & 5.50 & Loamy sand & 14.43 & 6.28 & 8.15 & 36.52 & 0.95 & 8.20 & 19.5 & 7.52 & 0.42 & 3.43 \\
\hline & $120-150$ & 4.20 & 87.00 & 10.00 & 3.00 & Sand & 12.62 & 4.82 & 7.80 & 32.98 & 0.81 & 8.23 & 35.4 & 7.45 & 0.34 & 1.70 \\
\hline \multirow[t]{3}{*}{12} & $0-18$ & 6.90 & 84.50 & 10.00 & 5.50 & Loamy sand & 14.43 & 6.28 & 8.15 & 36.52 & 3.83 & 8.18 & 26.5 & 8.85 & 0.61 & 3.96 \\
\hline & 18-68 & 6.70 & 84.50 & 7.50 & 8.00 & Loamy sand & 15.58 & 7.57 & 8.01 & 38.60 & 12.21 & 7.93 & 22.5 & 12.74 & 0.49 & 5.12 \\
\hline & 68-120+ & 0.00 & 82.00 & 7.50 & 10.50 & Loamy sand & 17.00 & 8.87 & 8.14 & 40.28 & 1.18 & 8.33 & 22.2 & 7.62 & 0.39 & 6.34 \\
\hline \multirow[t]{4}{*}{13} & 0-30 & 0.00 & 74.50 & 15.00 & 10.50 & Sandy loam & 18.19 & 8.97 & 9.21 & 40.86 & 5.35 & 7.95 & 20.1 & 9.56 & 0.78 & 7.43 \\
\hline & $30-60$ & 10.30 & 74.50 & 12.50 & 13.00 & Sandy loam & 18.95 & 10.08 & 8.87 & 42.01 & 6.12 & 8.03 & 21.5 & 9.92 & 0.62 & 8.49 \\
\hline & $60-95$ & 0.00 & 77.00 & 15.00 & 8.00 & Sandy loam & 16.82 & 7.78 & 9.05 & 39.14 & 6.73 & 8.07 & 20.3 & 10.20 & 0.43 & 4.95 \\
\hline & 95-130 & 42.10 & 79.50 & 12.50 & 8.00 & Loamy sand & 20.12 & 12.37 & 7.75 & 43.45 & 1.14 & 8.14 & 21.9 & 7.61 & 0.26 & 4.48 \\
\hline & & & & & & Piedmo & t plain, & ummit & & & & & & & & \\
\hline 14 & $0-30$ & 2.70 & 84.50 & 5.00 & 10.50 & Loamy sand & 16.67 & 8.83 & 7.83 & 40.10 & 0.86 & 8.21 & 60.3 & 7.48 & 0.49 & 6.62 \\
\hline 15 & 0-10 & 19.40 & 84.50 & 10.00 & 5.50 & Loamy sand & 14.43 & 6.28 & 8.15 & 36.52 & 5.48 & 8.05 & 36.4 & 9.62 & 0.77 & 4.41 \\
\hline & $10-45$ & 9.00 & 87.00 & 7.50 & 5.50 & Loamy sand & 13.97 & 6.17 & 7.80 & 36.34 & 8.12 & 8.16 & 44.7 & 10.84 & 0.77 & 4.41 \\
\hline & $45-70$ & 0.00 & 79.50 & 10.00 & 10.50 & Sandy loam & 17.36 & 8.90 & 8.46 & 40.47 & 6.73 & 8.17 & 38.1 & 10.20 & 0.26 & 5.98 \\
\hline 16 & $0-13$ & 22.20 & 89.50 & 7.50 & 3.00 & Sand & 12.07 & 4.66 & 7.42 & 32.80 & 0.44 & 8.29 & 26.5 & 7.28 & 0.98 & 3.49 \\
\hline & & & & & & Tableland & Drainag & chann & & & & & & & & \\
\hline 17 & 0-18 & 0.00 & 94.50 & 5.00 & 0.50 & Sand & 9.15 & 2.88 & 6.27 & 22.51 & 0.42 & 8.20 & 26.3 & 7.27 & 1.12 & 2.39 \\
\hline & $18-50$ & 0.00 & 97.00 & 2.50 & 0.50 & Sand & 8.55 & 2.70 & 5.85 & 22.33 & 0.28 & 8.24 & 22.2 & 7.21 & 0.87 & 1.69 \\
\hline & $50-80$ & 0.00 & 92.00 & 7.50 & 0.50 & Sand & 9.78 & 3.07 & 6.71 & 22.69 & 0.25 & 8.28 & 21.7 & 7.19 & 0.65 & 1.07 \\
\hline & $80-120$ & 7.40 & 87.00 & 7.50 & 5.50 & Loamy sand & 13.97 & 6.17 & 7.80 & 36.34 & 0.58 & 8.45 & 21.9 & 7.35 & 0.69 & 4.18 \\
\hline & $120-150$ & 0.00 & 82.00 & 10.00 & 8.00 & Loamy sand & 15.98 & 7.64 & 8.34 & 38.78 & 1.41 & 7.87 & 32.7 & 7.73 & 0.52 & 5.21 \\
\hline 18 & $0-15$ & 0.00 & 94.50 & 5.00 & 0.50 & Sand & 9.15 & 2.88 & 6.27 & 22.51 & 0.43 & 7.91 & 20.7 & 7.28 & 0.85 & 1.63 \\
\hline & $15-45$ & 0.00 & 97.00 & 2.50 & 0.50 & Sand & 8.55 & 2.70 & 5.85 & 22.33 & 0.18 & 8.19 & 21.5 & 7.16 & 0.85 & 1.63 \\
\hline & $45-95$ & 6.30 & 84.50 & 10.00 & 5.50 & Loamy sand & 14.43 & 6.28 & 8.15 & 36.52 & 0.19 & 8.26 & 17.0 & 7.17 & 0.71 & 4.24 \\
\hline & 95-125+ & 6.30 & 89.50 & 7.50 & 3.00 & Sand & 12.07 & 4.66 & 7.42 & 32.80 & 0.20 & 8.24 & 12.8 & 7.17 & 0.26 & 1.48 \\
\hline 19 & $0-16$ & 0.00 & 94.50 & 5.00 & 0.50 & Sand & 9.15 & 2.88 & 6.27 & 22.51 & 0.43 & 7.91 & 20.7 & 7.28 & 0.85 & 1.63 \\
\hline & $16-50$ & 0.00 & 97.00 & 2.50 & 0.50 & Sand & 8.55 & 2.70 & 5.85 & 22.33 & 0.18 & 8.19 & 21.5 & 7.16 & 0.85 & 1.63 \\
\hline & $50-100$ & 6.40 & 84.50 & 10.00 & 5.50 & Loamy sand & 14.43 & 6.28 & 8.15 & 36.52 & 0.19 & 8.26 & 17.0 & 7.17 & 0.71 & 4.24 \\
\hline & $100-130+$ & 6.40 & 89.50 & 7.50 & 3.00 & Sand & 12.07 & 4.66 & 7.42 & 32.80 & 0.20 & 8.24 & 12.8 & 7.17 & 0.26 & 1.48 \\
\hline 20 & $0-18$ & 0.00 & 94.50 & 5.00 & 0.5 & sand & 9.15 & 2.88 & 6.27 & 22.51 & 0.43 & 7.91 & 20.7 & 7.28 & 0.85 & 1.63 \\
\hline & $18-55$ & 0.00 & 97.00 & 2.50 & 0.5 & sand & 8.55 & 2.70 & 5.85 & 22.33 & 0.18 & 8.19 & 21.5 & 7.16 & 0.85 & 1.63 \\
\hline & $55-105$ & 6.20 & 84.50 & 10.00 & 5.5 & Loamy sand & 14.43 & 6.28 & 8.15 & 36.52 & 0.19 & 8.26 & 17.0 & 7.17 & 0.71 & 4.24 \\
\hline & $105-130+$ & 6.20 & 89.50 & 7.50 & 3.00 & Sand & 12.07 & 4.66 & 7.42 & 32.80 & 0.20 & 8.24 & 12.8 & 7.17 & 0.26 & 1.48 \\
\hline 21 & 0-20 & 5.30 & 92.00 & 5.00 & 3.00 & Sand & 11.55 & 4.51 & 7.04 & 32.62 & 0.31 & 8.20 & 20.9 & 7.22 & 0.87 & 3.19 \\
\hline & $20-70$ & 7.10 & 82.00 & 10.00 & 8.00 & Loamy sand & 15.98 & 7.64 & 8.34 & 38.78 & 0.37 & 8.16 & 17.6 & 7.25 & 0.75 & 5.85 \\
\hline & $70-120$ & 14.30 & 82.00 & 10.00 & 8.00 & Loamy sand & 15.98 & 7.64 & 8.34 & 38.78 & 0.39 & 8.22 & 18.6 & 7.26 & 0.70 & 5.71 \\
\hline & $120-150$ & 7.50 & 79.50 & 12.50 & 8.00 & Loamy sand & 16.39 & 7.71 & 8.69 & 38.96 & 0.41 & 8.22 & 16.4 & 7.27 & 0.68 & 5.65 \\
\hline 22 & $0-20$ & 5.30 & 92.00 & 5.00 & 3.00 & Sand & 11.55 & 4.51 & 7.04 & 32.62 & 0.31 & 8.20 & 20.9 & 7.22 & 0.87 & 3.19 \\
\hline & $20-70$ & 7.10 & 82.00 & 10.00 & 8.00 & Loamy sand & 15.98 & 7.64 & 8.34 & 38.78 & 0.37 & 8.16 & 17.6 & 7.25 & 0.75 & 5.85 \\
\hline & $70-120$ & 14.30 & 82.00 & 10.00 & 8.00 & Loamy sand & 15.98 & 7.64 & 8.34 & 38.78 & 0.39 & 8.22 & 18.6 & 7.26 & 0.70 & 5.71 \\
\hline & $120-150$ & 7.50 & 79.50 & 12.50 & 8.00 & Loamy sand & 16.39 & 7.71 & 8.69 & 38.96 & 0.41 & 8.22 & 16.4 & 7.27 & 0.68 & 5.65 \\
\hline
\end{tabular}


Continue.Table 1.

\begin{tabular}{|c|c|c|c|c|c|c|c|c|c|c|c|c|c|c|c|c|}
\hline \multirow[t]{2}{*}{$\begin{array}{c}\text { Soil } \\
\text { Profile }\end{array}$} & \multirow[t]{2}{*}{$\begin{array}{c}\text { Depth } \\
\text { (cm) }\end{array}$} & \multirow[t]{2}{*}{$\begin{array}{c}\text { Gravels } \\
\%\end{array}$} & \multicolumn{3}{|c|}{$\begin{array}{c}\text { Particles size } \\
\text { distribution }\end{array}$} & \multirow[t]{2}{*}{ Texture } & \multirow[t]{2}{*}{$\begin{array}{c}\text { F.C } \\
\%\end{array}$} & \multirow[t]{2}{*}{$\begin{array}{c}\text { W.P } \\
\%\end{array}$} & \multirow[t]{2}{*}{$\begin{array}{c}\text { A.W } \\
\%\end{array}$} & \multirow[t]{2}{*}{$\begin{array}{c}\text { S.P } \\
\%\end{array}$} & \multirow[t]{2}{*}{$\begin{array}{c}\text { EC } \\
\mathrm{dS} / \mathrm{m}\end{array}$} & \multirow[t]{2}{*}{ pH } & \multirow[t]{2}{*}{$\begin{array}{c}\mathrm{CaCO}_{3} \\
\%\end{array}$} & \multirow[t]{2}{*}{ SAR } & \multirow[t]{2}{*}{$\begin{array}{c}\mathrm{OM} \\
\%\end{array}$} & \multirow[t]{2}{*}{$\begin{array}{c}\text { CEC } \\
\mathrm{meq} / 100 \mathrm{~g}\end{array}$} \\
\hline & & & Sand & silt & clay & & & & & & & & & & & \\
\hline \multirow[t]{5}{*}{23} & $0-14$ & 0.00 & 84.50 & 7.50 & 8.00 & Loamy sand & 15.58 & 7.57 & 8.01 & 38.60 & 0.31 & 8.12 & 26.9 & 7.22 & 0.78 & 5.93 \\
\hline & 14-50 & 8.00 & 82.00 & 7.50 & 10.50 & Loamy sand & 17.00 & 8.87 & 8.14 & 40.28 & 0.30 & 8.35 & 21.9 & 7.22 & 0.69 & 7.18 \\
\hline & $50-75$ & 0.00 & 79.50 & 7.50 & 13.00 & Sandy loam & 18.30 & 10.07 & 8.22 & 41.65 & 0.35 & 8.20 & 24 & 7.24 & 0.59 & 8.40 \\
\hline & $75-100$ & 0.00 & 77.00 & 7.50 & 15.50 & Sandy loam & 19.51 & 11.21 & 8.29 & 42.81 & 0.27 & 8.23 & 20.9 & 7.20 & 0.69 & 10.18 \\
\hline & $100-150$ & 0.00 & 77.00 & 10.00 & 13.00 & Sandy loam & 18.62 & 10.08 & 8.54 & 41.83 & 0.22 & 8.37 & 20.3 & 7.18 & 0.42 & 7.93 \\
\hline \multirow[t]{4}{*}{24} & $0-18$ & 0.00 & 94.50 & 5.00 & 0.50 & Sand & 9.15 & 2.88 & 6.27 & 22.51 & 0.42 & 8.20 & 26.3 & 7.27 & 1.12 & 2.39 \\
\hline & $18-75$ & 0.00 & 97.00 & 2.50 & 0.50 & Sand & 8.55 & 2.70 & 5.85 & 22.33 & 0.28 & 8.24 & 22.2 & 7.21 & 0.87 & 1.69 \\
\hline & $75-120$ & 0.00 & 92.00 & 7.50 & 0.50 & Sand & 9.78 & 3.07 & 6.71 & 22.69 & 0.25 & 8.28 & 21.7 & 7.19 & 0.65 & 1.07 \\
\hline & $75-120$ & 0.00 & 92.00 & 7.50 & 0.50 & Sand & 9.78 & 3.07 & 6.71 & 22.69 & 0.25 & 8.28 & 21.7 & 7.19 & 0.65 & 1.07 \\
\hline \multicolumn{17}{|c|}{ Tableland, Summit } \\
\hline \multirow[t]{2}{*}{25} & $0-35$ & 0.00 & 94.50 & 5.00 & 0.50 & Sand & 9.15 & 2.88 & 6.27 & 22.51 & 0.43 & 7.91 & 20.7 & 7.28 & 0.85 & 1.63 \\
\hline & $35-80$ & 0.00 & 97.00 & 2.50 & 0.50 & Sand & 8.55 & 2.70 & 5.85 & 22.33 & 0.18 & 8.19 & 21.5 & 7.16 & 0.85 & 1.63 \\
\hline 26 & $0-50$ & 5.90 & 82.00 & 12.50 & 5.50 & Loamy sand & 14.91 & 6.39 & 8.52 & 36.70 & 2.12 & 8.40 & 32.1 & 8.06 & 0.79 & 4.46 \\
\hline 27 & $0-20$ & 0.00 & 87.00 & 10.00 & 3.00 & Sand & 12.62 & 4.82 & 7.80 & 32.98 & 0.92 & 8.18 & 21.1 & 7.50 & 0.69 & 2.68 \\
\hline & $20-35$ & 0.00 & 82.00 & 7.50 & 10.50 & Loamy sand & 17.00 & 8.87 & 8.14 & 40.28 & 1.32 & 8.41 & 41.6 & 7.69 & 0.47 & 6.57 \\
\hline & $35-80$ & 0.00 & 82.00 & 10.00 & 8.00 & Loamy sand & 15.98 & 7.64 & 8.34 & 38.78 & 1.73 & 8.38 & 54.2 & 7.88 & 0.21 & 4.34 \\
\hline 28 & $0-40$ & 0.00 & 79.50 & 12.50 & 8.00 & Loamy sand & 16.39 & 7.71 & 8.69 & 38.96 & 1.72 & 8.66 & 32.7 & 7.88 & 0.27 & 4.51 \\
\hline 29 & $0-50$ & 6.30 & 79.50 & 10.00 & 10.50 & Sandy loam & 17.36 & 8.90 & 8.46 & 40.47 & 4.62 & 8.20 & 43.5 & 9.22 & 0.22 & 5.87 \\
\hline 30 & $0-18$ & 0.00 & 89.50 & 5.00 & 5.50 & Sand & 13.52 & 6.06 & 7.46 & 36.16 & 4.47 & 8.20 & 52.2 & 9.15 & 1.02 & 5.11 \\
\hline & $18-55$ & 0.00 & $\mathbf{7 4 . 5 0}$ & $\mathbf{7 . 5 0}$ & 18.00 & sandy loam & 20.66 & 12.30 & 8.36 & 43.82 & 5.43 & 8.34 & 45.6 & 9.60 & 0.77 & 11.91 \\
\hline 31 & $0-18$ & 0.00 & 89.50 & 5.00 & 5.50 & sand & 13.52 & 6.06 & $\mathbf{7 . 4 6}$ & 36.16 & 4.47 & 8.20 & 52.2 & 9.15 & 1.02 & 5.11 \\
\hline & $18-55$ & 0.00 & $\mathbf{7 4 . 5 0}$ & $\mathbf{7 . 5 0}$ & 18.00 & Sandy loam & 20.66 & 12.30 & 8.36 & 43.82 & 5.43 & 8.34 & 45.6 & 9.60 & 0.77 & 11.91 \\
\hline 32 & $0-20$ & 0.00 & 89.50 & 7.50 & 3.00 & Sand & 12.07 & 4.66 & 7.42 & 32.80 & 0.75 & 8.30 & 48.4 & 7.43 & 0.88 & 3.21 \\
\hline & $20-70$ & 2.40 & 84.50 & $\mathbf{7 . 5 0}$ & 8.00 & Loamy sand & 15.58 & 7.57 & 8.01 & 38.60 & 0.52 & 8.40 & 29.6 & 7.32 & 0.67 & 5.63 \\
\hline 33 & $0-50$ & 7.00 & 82.00 & 10.00 & 8.00 & Loamy sand & 15.98 & $\mathbf{7 . 6 4}$ & 8.34 & 38.78 & 1.03 & 8.36 & 30.6 & 7.55 & 0.36 & 4.76 \\
\hline 34 & $0-25$ & 4.50 & 89.50 & 7.50 & 3.00 & Sand & 12.07 & 4.66 & 7.42 & 32.80 & 0.32 & 8.30 & 28.2 & 7.23 & 1.05 & 3.69 \\
\hline & $25-50$ & 0.00 & 87.00 & 12.50 & 0.50 & Sand & 11.12 & 3.47 & 7.64 & 23.05 & 0.39 & 8.30 & 37.7 & 7.26 & 0.69 & 1.18 \\
\hline 35 & $0-30$ & 6.70 & $\mathbf{7 4 . 5 0}$ & 12.50 & 13.00 & Sandy loam & 18.95 & 10.08 & 8.87 & 42.01 & 0.47 & 8.20 & 24.0 & 7.30 & 1.29 & 10.36 \\
\hline & $30-60$ & 4.20 & 72.00 & 10.00 & 18.00 & Sandy loam & 20.95 & 12.27 & 8.69 & 44.00 & 1.08 & 8.28 & 25.9 & 7.58 & 0.59 & 11.40 \\
\hline & $60-100$ & 4.20 & 69.50 & 15.00 & 15.50 & Sandy loam & 20.45 & 11.16 & 9.29 & 43.35 & 1.30 & 8.48 & 25.5 & 7.68 & 0.41 & 9.40 \\
\hline & $100-150$ & 0.00 & 72.00 & 12.50 & 15.50 & Sandy loam & 20.12 & 11.17 & 8.95 & 43.17 & 1.13 & 8.53 & 23.2 & $\mathbf{7 . 6 0}$ & 0.40 & 9.37 \\
\hline 36 & $0-30$ & 8.50 & 87.00 & 5.00 & 8.00 & Loamy sand & 15.20 & 7.50 & 7.70 & 38.42 & 0.41 & 8.44 & 24.4 & 7.27 & 0.70 & 5.71 \\
\hline & $30-60$ & 5.60 & 84.50 & 7.50 & 8.00 & Loamy sand & 15.58 & 7.57 & 8.01 & 38.60 & 1.21 & 8.57 & 24.8 & 7.64 & 0.70 & 5.71 \\
\hline & $60-100$ & 7.10 & 74.50 & 15.00 & 10.50 & Sandy loam & 18.11 & 8.96 & 9.14 & 40.83 & 3.09 & 8.30 & 21.1 & 8.51 & $\mathbf{0 . 3 0}$ & 6.09 \\
\hline & $100-150$ & 7.90 & 77.00 & 10.00 & 13.00 & Sandy loam & 18.62 & 10.08 & 8.54 & 41.83 & 3.79 & 8.28 & 27.7 & 8.84 & 0.26 & 7.48 \\
\hline 37 & $0-40$ & 9.00 & $\mathbf{7 4 . 5 0}$ & 15.00 & 10.50 & Sandy loam & 18.11 & 8.96 & 9.14 & 40.83 & 7.15 & 8.20 & 30.6 & 10.39 & 0.45 & 6.51 \\
\hline 38 & $0-16$ & 7.00 & 82.00 & 10.00 & 8.00 & Loamy sand & 15.98 & 7.64 & 8.34 & 38.78 & 1.03 & 8.36 & 30.6 & 7.55 & 0.36 & 4.76 \\
\hline 39 & $0-30$ & 0.00 & 82.00 & 10.00 & 8.00 & Loamy sand & 15.98 & 7.64 & 8.34 & 38.78 & 4.24 & 8.34 & 56.5 & 9.04 & 0.29 & 4.56 \\
\hline & 30-57 & 6.30 & $\mathbf{7 9 . 5 0}$ & 10.00 & 10.50 & Sandy loam & 17.36 & 8.90 & 8.46 & 40.47 & 4.62 & 8.20 & 43.5 & 9.22 & 0.22 & 5.87 \\
\hline 40 & $0-22$ & 8.70 & 84.50 & 10.00 & 5.50 & Loamy sand & 14.43 & 6.28 & 8.15 & 36.52 & 0.31 & 8.36 & 32.3 & 7.22 & 0.92 & 4.83 \\
\hline & $22-40$ & 2.40 & 87.00 & 10.00 & 3.00 & Sand & 12.62 & 4.82 & $\mathbf{7 . 8 0}$ & 32.98 & 0.29 & 8.85 & 46.4 & 7.21 & 0.41 & 1.90 \\
\hline 41 & $0-18$ & 8.70 & 84.50 & 10.00 & 5.50 & Loamy sand & 14.43 & 6.28 & 8.15 & 36.52 & 0.45 & $\mathbf{7 . 9 8}$ & 33.7 & 7.29 & 0.95 & 4.91 \\
\hline
\end{tabular}


subunits, namely summit and drainage channels. The surface of these subunits mainly rocky that is consists of Marmarica limestone.

\section{Tableland}

The tableland constitutes a prominent geomorphologic unit bounding the piedmont plain from the south and covers an area 1685.8 faddan. it occupies the northern extermely of the great Marmarica Homoclinal plateau and extend to Qattara depression. The northern portion of the table land is dissected by a number of short and deep consequent wadis acting during rainy season as active drainage lines. The bottom of the drainage lines is occupied by thick alluvial deposits composed of gravel, cobbles intermixed with sand, silt, and clay. These drainage lines or channel dissecting the tableland and the other landforms except plateau escarpment are mainly cultivated by orchards tress such as olive and fig which are dominant. There are also some scattered pomegranate and almond counted on fingers. The surface of this unit especially the summit is covered by thick sediments and it is cultivated by barley as rainfed agricultural system. Accordingly, this unit is subdivided into two subunits, namely drainage channel and summit.

\section{Tableland drainage channel}

The drainage channel dissecting tableland covers an area 77.7 faddan and are represented by 8 soil profiles. The soil of the drainage channel of this landform are deep (soil depth $>100 \mathrm{~cm}$ ). the gravel content is less than $15 \%$ so it is not affected the soil texture which is described in general as coarse-texture except the three subsurface layers of soil profile 23 where the soil texture is moderately coarse-texture soils. Related to the soil texture, the soil water characteristics especially the available water is very poor. Based on the data tabulated in Table (1) and related to electrical conductivity, $\mathrm{pH}$, and SAR, the soils of this subunit is fresh soils due to the washed process by flood water comparable to the same subunit in piedmont plain. As mentioned in the previous landforms, the soil fertility if this unit is also poor.

\section{Tableland summit}

This area covers an area of about 1608,2 faddan and were represent by 28 soil profiles 11 out of them are characterized by rock exposures. The soil depth of this unit as shown in table (1) ranges from very shallow (less than $25 \mathrm{~cm}$ ) to deep (more than $100 \mathrm{~cm}$ ). The soil gravel is in general less than $15 \%$ so it does not modified the soil texture which ranges from coarse-texture to moderately coarse-texture. Consequently, the soil water characteristics are very poor. In general, the soils of this subunit are fresh soils where EC is less than $4 \mathrm{dS} / \mathrm{m}, \mathrm{pH}$ is less than 8.5, and SAR is less than 13. The exceptional cases were appeared in the soils of profiles $29,30,31,37$, and 39, respectively, where the soils of these profiles are saline soils. The calcium carbonate content range from strongly to extermely calcareous (20.7 to $56.5 \%)$. As for the soil fertility status of this landform subunit measured by CEC and OM, the soils of this unit is very poor.

\section{Landuse and land cover (LULC) and accuracy assessment}

The NDVI of the image captured in August 28, 2017 was processed for assessing the LULC of the study area. NDVI image was resulted as shown in Figure (4) and reclassified into 4 classes taking the field truth in consideration. The resulted classes were named as water body (class 1), cultivated land (class 2), Bare land (class 3 ), and low dense rangeland (class 4). The accuracy assessment of the NDVI image was measured using statistical pivot table. As shown in Table (2), the overall accuracy resulted from the NDVI image was $71.88 \%$. However, the producer's accuracy for the LULC classes were $100,57.5,92.5,37.5 \%$ for water body, cultivated land, Bare land, and low dense rangeland, respectively. On contrary, the user's accuracy attained 100, 95.83, 54.41, and $53.57 \%$ for the same LULC classes. By running the supervised classification as tool for identifying the LULC classes dominant in the study area, Figure (4), it was found that this tool is more accurate comparing with NDVI process whereas the overall accuracy attained $81.88 \%$. This classification showed that as shown in Table (2) the producer's accuracy for the LULC classes were 100, 90, 77.5, $60 \%$ for water body, cultivated land, Bare land, and low dense rangeland, respectively. On contrary, the user's accuracy attained 100, 87.80, 65.96, $75 \%$ for the same LULC classes. This variance between the used methods may be attributed to the long distance between orchard trees (more than $10 \mathrm{~m}$ ), so the NDVI considered the uncovered area among trees as bare land while it was recognized as cultivated land throughout digitizing the sampling area by producer during supervised classification. The processed satellite image was captured in August 28, 2017, where the area was uncovered by field crops (barley), so NDVI process assigned them as bare land while it was assigned as cultivated land during supervised classification. In sustainable landuse, the LULC of the studied area contributed in define the more appropriate location that suitable for agriculture processes and which area is planted by which types of crops. In studied area condition as well as the area extended from Ras El Hekma to El Salloum, it was found that wadi streams and deltas are suitable for orchard trees where it is 
Table 2. Accuracy assessment resulted from Supervised Classification and Normalized Difference Vegetation Index (NDVI)

\begin{tabular}{|c|c|c|c|c|c|c|c|c|}
\hline \multicolumn{9}{|c|}{ Accuracy assessment matrix of LULC resulted from Supervised classification } \\
\hline \multirow{2}{*}{\multicolumn{2}{|c|}{ LULC classes }} & \multicolumn{5}{|c|}{ Field Truth } & \multirow{2}{*}{$\begin{array}{c}\text { User's } \\
\text { Accuracy }\end{array}$} & \multirow{2}{*}{$\begin{array}{c}\text { Omission } \\
\text { error }\end{array}$} \\
\hline & & Water body & Cultivated land & Bare land & Low dense rangeland & Total & & \\
\hline \multirow{5}{*}{ 苋 } & Water body & 40 & $\mathbf{0}$ & $\mathbf{0}$ & $\mathbf{0}$ & 40 & 100.00 & $\mathbf{0}$ \\
\hline & Cultivated land & $\mathbf{0}$ & 36 & 1 & 4 & 41 & 87.80 & 5.69 \\
\hline & Bare land & $\mathbf{0}$ & 4 & 31 & 12 & 47 & 65.96 & 24.26 \\
\hline & Low dense rangeland & $\mathbf{0}$ & $\mathbf{0}$ & 8 & 24 & 32 & 75.00 & 10.67 \\
\hline & Total & 40 & 40 & 40 & 40 & 160 & & \\
\hline & Producer's Accuracy & 100 & 90 & 77.5 & 60 & & \multicolumn{2}{|c|}{ Overall Accuracy } \\
\hline & Omission error & $\mathbf{0}$ & 4.44 & 11.61 & 26.67 & & \multicolumn{2}{|c|}{131} \\
\hline & & & & & & & \multicolumn{2}{|c|}{81.88} \\
\hline \multicolumn{9}{|c|}{ Accuracy assessment matrix of LULC resulted from NDVI } \\
\hline \multirow{10}{*}{ صِّ } & & & & Field Truth & & & & Omission \\
\hline & LULC classes & Water body & Cultivated land & Bare land & Low dense rangeland & Total & $\begin{array}{c}\text { User's } \\
\text { Accuracy }\end{array}$ & error \\
\hline & Water body & 40 & $\mathbf{0}$ & $\mathbf{0}$ & $\mathbf{0}$ & 40 & 100.00 & $\mathbf{0}$ \\
\hline & Cultivated land & $\mathbf{0}$ & 23 & $\mathbf{0}$ & 1 & 24 & 95.83 & 1.04 \\
\hline & Bare land & $\mathbf{0}$ & 7 & 37 & 24 & 68 & 54.41 & 56.97 \\
\hline & Low dense rangeland & $\mathbf{0}$ & 10 & 3 & 15 & 28 & 53.57 & 24.27 \\
\hline & Total & 40 & 40 & 40 & 40 & 160 & & \\
\hline & Producer's Accuracy & 100 & $\mathbf{5 7 . 5}$ & 92.5 & 37.5 & & \multicolumn{2}{|c|}{ Overall Accuracy } \\
\hline & Omission error & $\mathbf{0}$ & 29.57 & 3.24 & 66.67 & & \multicolumn{2}{|c|}{115} \\
\hline & & & & & & & \multicolumn{2}{|c|}{71.88} \\
\hline
\end{tabular}




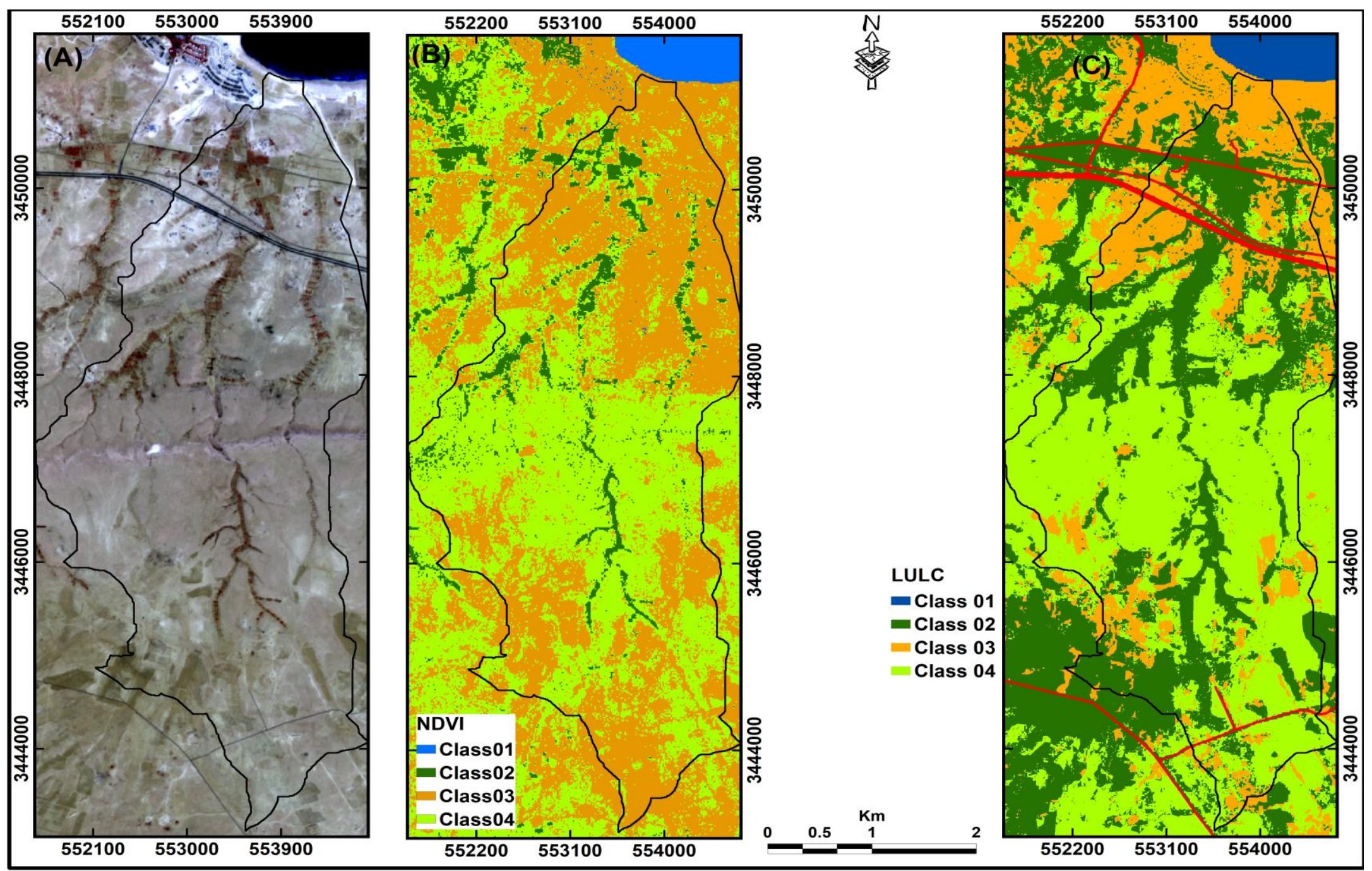

Figure 4. Raw data of the sentinel 2A image (A), NDVI classification (B), Supervised classification (C) Class 01: Water body, Class 02: Cultivated land, class 03: Bare land, class 04: Low dense rangeland 
Table 3. Soil characteristics of the dominant soil mapping and their suitability classes for the selected crops

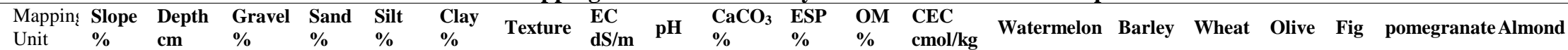

Coastal Plain (24 faddan)

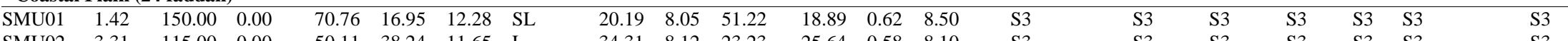

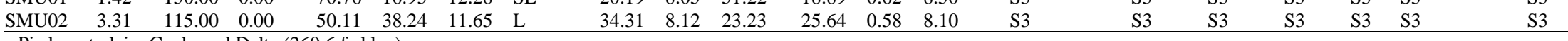

Piedmont plain, Coalesced Delta (269.6 faddan)

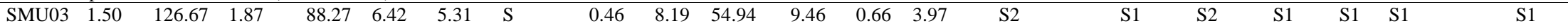

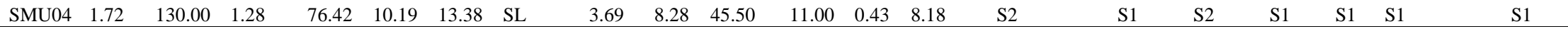

Piedmont plain, Drainage Channel (388 faddan)

$\begin{array}{llllllllllllllllllllllllll}\text { SMU03 } & 1.90 & 150.00 & 3.29 & 83.25 & 10.50 & 6.25 & \text { LS } & 4.32 & 8.03 & 22.15 & 11.09 & 0.42 & 3.87 & \text { S2 } & \text { S1 } & \text { S1 } & \text { S1 } & \text { S1 } & \text { S1 } & \text { S1 }\end{array}$

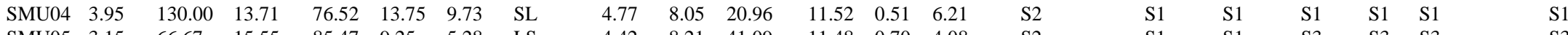

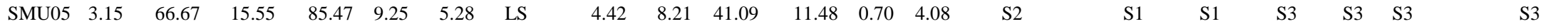

Piedmont plain, Summit

$\begin{array}{lllllllllllllllllllll}\text { SMU05 } & 3.16 & 70 & 7.27 & 83.96 & 8.75 & 7.29 & \text { LS } & 7.25 & 8.15 & 41.16 & 12.70 & 0.59 & 4.97 & \text { S2 } & \text { S2 } & \text { S2 } & \text { S3 } & \text { S3 } & \text { S3 } & \text { S3 }\end{array}$

$\begin{array}{lllllllllllllllllllllll}\text { SMU06 } & 1.59 & 30 & 2.70 & 84.50 & 5.00 & 10.50 & \text { LS } & 0.86 & 8.21 & 60.30 & 9.65 & 0.49 & 6.62 & \mathrm{~S} 3 & & \mathrm{~S} 3 & \mathrm{~S} 3 & \mathrm{~N} & \mathrm{~N} & \mathrm{~N} & & \mathrm{~N}\end{array}$

$\begin{array}{lllllllllllllllllllllllll}\text { SMU07 } & 3.15 & 13 & 22.20 & 89.50 & 7.50 & 3.00 & \mathrm{~S} & 0.44 & 8.29 & 26.50 & 9.45 & 0.98 & 3.49 & \mathrm{~S} 3 & \mathrm{~N} 3 & \mathrm{~S} 3 & \mathrm{~N} & \mathrm{~N} & \mathrm{~N} & \mathrm{~N}\end{array}$

Tableland, Drainage channel

$\begin{array}{lllllllllllllllllllllll}\text { SMU03 } & 1.80 & 137.50 & 4.71 & 88.46 & 7.46 & 4.08 & \text { S } & 0.35 & 8.21 & 19.83 & 11.64 & 0.73 & 3.44 & \text { S2 } & & \text { S1 } & \text { S2 } & \text { S1 } & \text { S1 } & \text { S1 } & \text { S1 } & \text { S }\end{array}$

$\begin{array}{lllllllllllllllllllll}\text { SMU04 } & 2.17 & 150.00 & 1.92 & 79.32 & 8.33 & 12.35 & \text { SL } & 0.28 & 8.29 & 22.02 & 11.60 & 0.59 & 8.02 & \text { S2 } & \text { S1 } & \text { S2 } & \text { S1 } & \text { S1 } & \text { S1 } & \text { S1 }\end{array}$

Tableland, Summit

\begin{tabular}{lllllllllllllll}
\hline SMU04 & 0.98 & 150.00 & 5.32 & 75.83 & 11.25 & 12.92 & SL & 1.72 & 8.39 & 24.61 & 10.06 & 0.53 & 8.19 & S2
\end{tabular}

$\begin{array}{lllllllllllllll}\text { SMU05 } & 1.50 & 62.43 & 2.84 & 85.45 & 9.02 & 5.53 & \text { LS } & 1.46 & 8.30 & 35.10 & 9.94 & 0.61 & 3.96 & \text { S2 }\end{array}$

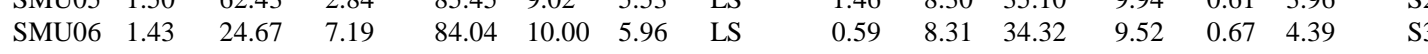

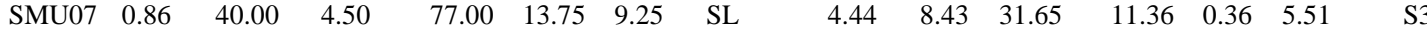

SMU08 $1.22 \quad 53.33 \quad 2.10 \quad 79.44 \quad 7.79 \quad 12.77 \quad$ SL

SMU01: Slightly slope, deep, moderately coarse-texture soils

SMU03: Slightly slope, deep, coarse-texture soils

$\begin{array}{lllllll}4.95 & 8.26 & 46.34 & 11.61 & 0.64 & 8.41 & \mathrm{~S} 2\end{array}$

SMU02: Slightly slope, deep, medium-texture soils

SMU05: Slightly slope, moderately deep, coarse-texture soils

SMU04: Slightly slope, deep, moderately coarse-texture soils

SMU07: Slightly slope, shallow, moderately coarse-texture soils

: Slightly slope, shallow, coarse-texture soils

SMU08: Slightly slope, moderately deep, moderately coarse-texture soils 
Table 4. Soil mapping, Landform, and crop types matrix showing soil suitability classes and landform preference

\begin{tabular}{|c|c|c|c|c|c|c|c|c|c|c|}
\hline Landform & Landform subunit & Crop & SMU01 & SMU02 & SMU03 & SMU04 & SMU05 & SMU06 & SMU07 & SMU08 \\
\hline \multirow{7}{*}{\multicolumn{2}{|c|}{ Coastal Plain }} & Watermelon & $\mathrm{S}^{\mathrm{P} 3}$ & $\mathrm{~S}^{\mathrm{P} 3}$ & 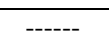 & ----- & 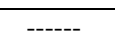 & ----- & $\begin{array}{ll}----- \\
--1\end{array}$ & ------ \\
\hline & & Barley & $\mathrm{S} 3^{\mathrm{P} 1}$ & $\mathrm{~S} 3^{\mathrm{P} 1}$ & ------ & ----- & ------ & ------ & ------ & ------ \\
\hline & & Wheat & $\mathrm{S} 3^{\mathrm{P} 2}$ & $\mathrm{~S} 3^{\mathrm{P} 2}$ & ----- & ----- & ----- & ----- & ----- & ------ \\
\hline & & Olives & $\mathrm{S} 3^{\mathrm{P} 3}$ & $\mathrm{~S} 3^{\mathrm{P} 3}$ & ----- & ----- & ----- & ----- & ----- & ------ \\
\hline & & Fig & $\mathrm{S} 3^{\mathrm{P} 3}$ & $\mathrm{~S} 3^{\mathrm{P} 3}$ & ----- & ----- & ----- & ---- & ---- & ----- \\
\hline & & pomegranate & $\mathrm{S}^{\mathrm{P} 3}$ & $\mathrm{~S} 3^{\mathrm{P} 3}$ & ---- & ----- & ---- & - & ---- & ------ \\
\hline & & Almond & $\mathrm{S} 3^{\mathrm{P} 3}$ & $\mathrm{~S} 3^{\mathrm{P} 3}$ & ----- & ----- & ----- & ----- & ----- & ------ \\
\hline \multirow{21}{*}{ Piedmont plain } & \multirow{7}{*}{ Coalesced Delta } & Watermelon & ----- & ----- & $\mathrm{S} 2^{\mathrm{P} 2}$ & $\mathrm{~S} 2^{\mathrm{P} 2}$ & ----- & $\begin{array}{l}----- \\
\end{array}$ & ----- & ------ \\
\hline & & Barley & ----- & ----- & $\mathrm{S} 1^{\mathrm{P} 1}$ & $\mathrm{~S} 1^{\mathrm{P} 1}$ & ----- & ----- & ----- & ------ \\
\hline & & Wheat & ------ & ------ & $\mathrm{S} 2^{\mathrm{P} 1}$ & $\mathrm{~S} 2^{\mathrm{P} 1}$ & ------ & ------ & ------ & ------ \\
\hline & & Olives & ------ & ------ & $\mathrm{S}^{\mathrm{P} 2}$ & $\mathrm{~S}^{\mathrm{P} 2}$ & ------ & ------ & ------ & ------ \\
\hline & & Fig & ------ & ------ & $\mathrm{S} 1^{\mathrm{P} 2}$ & $\mathrm{~S}^{\mathrm{P} 2}$ & ------ & ------ & ------ & ------ \\
\hline & & pomegranate & ----- & ------ & $\mathrm{S} 1^{\mathrm{P} 2}$ & $\mathrm{~S} 1^{\mathrm{P} 2}$ & -.---. & ------ & ------ & ------ \\
\hline & & Almond & ------ & ----- & $\mathrm{S} 1^{\mathrm{P} 2}$ & $\mathrm{~S} 1^{\mathrm{P} 2}$ & - & ----- & ----- & ------ \\
\hline & \multirow{7}{*}{ Drainage Channel } & Watermelon & $-\cdots$ & $-\cdots$ & $\mathrm{S} 2^{\mathrm{P} 1}$ & $\mathrm{~S} 2^{\mathrm{P} 1}$ & $\mathrm{~S} 2^{\mathrm{P1}}$ & $-\cdots$ & $-\cdots$ & ----- \\
\hline & & Barley & ----- & ------ & $\mathrm{S} 1^{\mathrm{P} 3}$ & $\mathrm{~S} 1^{\mathrm{P} 3}$ & $\mathrm{~S} 1^{\mathrm{P} 3}$ & ------ & ----- & ------ \\
\hline & & Wheat & ----- & ----- & $\mathrm{S} 1^{\mathrm{P} 3}$ & $\mathrm{~S} 1^{\mathrm{P} 3}$ & $\mathrm{~S} 1^{\mathrm{P} 3}$ & ----- & ----- & ------ \\
\hline & & Olives & ----- & ----- & $\mathrm{S} 1^{\mathrm{P} 1}$ & $\mathrm{~S} 2^{\mathrm{P} 1}$ & $\mathrm{~S} 3^{\mathrm{P} 1}$ & ------ & ------ & ------ \\
\hline & & Fig & ------ & ------ & $\mathrm{S} 1^{\mathrm{P} 1}$ & $\mathrm{~S} 2^{\mathrm{P} 1}$ & $\mathrm{~S} 3^{\mathrm{P} 1}$ & ------ & ------ & ------ \\
\hline & & pomegranate & ------ & ------ & $\mathrm{S} 1^{\mathrm{P} 1}$ & $\mathrm{~S} 2^{\mathrm{P} 1}$ & $\mathrm{~S} 3^{\mathrm{P} 1}$ & ------ & ------ & ------ \\
\hline & & Almond & ------ & ------ & $\mathrm{S} 1^{\mathrm{P} 1}$ & $\mathrm{~S} 2^{\mathrm{P} 1}$ & $\mathrm{~S} 3^{\mathrm{P} 1}$ & ----- & ------ & ------ \\
\hline & \multirow{7}{*}{ Summit } & Watermelon & ----- & ----- & - l & - n & $\mathrm{S} 2^{\mathrm{P} 2}$ & $\mathrm{~S} 3^{\mathrm{P} 2}$ & $\mathrm{~S} 3^{\mathrm{P} 2}$ & ---- \\
\hline & & Barley & ----- & ----- & ----- & ----- & $\mathrm{S} 2^{\mathrm{P} 1}$ & $\mathrm{~S} 3^{\mathrm{P} 1}$ & $\mathrm{~S} 3^{\mathrm{P} 1}$ & ------ \\
\hline & & Wheat & ------ & ------ & ----- & ----- & $\mathrm{S} 2^{\mathrm{P} 1}$ & $\mathrm{~S}^{\mathrm{P} 1}$ & $\mathrm{~S}^{\mathrm{P} 1}$ & ------ \\
\hline & & Olives & ---- & - n--- & - n - & ---- & $\mathrm{S} 3^{\mathrm{P} 3}$ & $\mathrm{~N}^{\mathrm{P} 3}$ & $\mathrm{~N}^{\mathrm{P3}}$ & ----- \\
\hline & & Fig & ---- & ----- & - & ----- & $\mathrm{S} 3^{\mathrm{P} 3}$ & $\mathrm{~N}^{\mathrm{P} 3}$ & $\mathrm{~N}^{\mathrm{P3}}$ & ----- \\
\hline & & pomegranate & ----- & ----- & ----- & ----- & $\mathrm{S} 3^{\mathrm{P} 3}$ & $\mathrm{~N}^{\mathrm{P3}}$ & $\mathrm{N}^{\mathrm{P3}}$ & ----- \\
\hline & & Almond & ------ & ------ & ------ & ------ & $\mathrm{S} 3^{\mathrm{P} 3}$ & $\mathrm{~N}^{\mathrm{P} 3}$ & $\mathrm{~N}^{\mathrm{P3}}$ & ------ \\
\hline \multirow{14}{*}{ Tableland } & \multirow{7}{*}{ Drainage channel } & Watermelon & ------ & ----- & $\mathrm{S} 2^{\mathrm{P} 1}$ & $\mathrm{~S} 2^{\mathrm{P} 1}$ & ----- & ----- & ----- & ------ \\
\hline & & Barley & ------ & ----- & $\mathrm{S} 1^{\mathrm{P} 3}$ & $\mathrm{~S} 1^{\mathrm{P} 3}$ & ------ & ------ & ------ & ------ \\
\hline & & Wheat & ------ & ------ & $\mathrm{S} 2^{\mathrm{P} 3}$ & $\mathrm{~S} 2^{\mathrm{P} 3}$ & ------ & ------ & ------ & ------ \\
\hline & & Olives & ------ & ------ & $\mathrm{S} 1^{\mathrm{P} 1}$ & $\mathrm{~S} 1^{\mathrm{P} 1}$ & ------ & ------ & ------ & ------ \\
\hline & & Fig & ----- & ------ & $\mathrm{S} 1^{\mathrm{P} 1}$ & $\mathrm{~S} 1^{\mathrm{P} 1}$ & ------ & ----- & ----- & ------ \\
\hline & & pomegranate & ------ & ----- & $\mathrm{S} 1^{\mathrm{P} 1}$ & $\mathrm{~S} 1^{\mathrm{P} 1}$ & ------ & ----- & ----- & ------ \\
\hline & & Almond & ----- & ------ & $\mathrm{S} 1^{\mathrm{P} 1}$ & $\mathrm{~S} 1^{\mathrm{P} 1}$ & ------ & ------ & ------ & ------ \\
\hline & \multirow{7}{*}{ Summit } & Watermelon & ------ & ------ & ----- & $\mathrm{S} 2^{\mathrm{P} 2}$ & $\mathrm{~S} 2^{\mathrm{P} 2}$ & $\mathrm{~S} 3^{\mathrm{P} 2}$ & $\mathrm{~S} 2^{\mathrm{P} 2}$ & $\mathrm{~S} 2^{\mathrm{P} 2}$ \\
\hline & & Barley & ------ & ------ & ----- & $\mathrm{S} 1^{\mathrm{P} 1}$ & $\mathrm{~S} 2^{\mathrm{P} 1}$ & $\mathrm{~S} 3^{\mathrm{P} 1}$ & $\mathrm{~S} 2^{\mathrm{P} 1}$ & $\mathrm{~S} 1^{\mathrm{P} 1}$ \\
\hline & & Wheat & ------ & ------ & ----- & $\mathrm{S} 2^{\mathrm{P} 1}$ & $\mathrm{~S} 2^{\mathrm{P} 1}$ & $\mathrm{~S} 3^{\mathrm{P} 1}$ & $\mathrm{~S} 2^{\mathrm{P} 1}$ & $\mathrm{~S} 2^{\mathrm{P} 1}$ \\
\hline & & Olives & --- & - & - & $\mathrm{S} 1^{\mathrm{P} 3}$ & $\mathrm{~S}^{\mathrm{P} 3}$ & $\mathrm{~N}^{\mathrm{P} 3}$ & $\mathrm{~N}^{\mathrm{P} 3}$ & $\mathrm{~S} 3^{\mathrm{P} 3}$ \\
\hline & & Fig & ---- & ----- & ---- & $\mathrm{S}^{\mathrm{P} 3}$ & $\mathrm{~S}^{\mathrm{P} 3}$ & $\mathrm{~N}^{\mathrm{P} 3}$ & $\mathrm{~N}^{\mathrm{P} 3}$ & $\mathrm{~S}^{\mathrm{P} 3}$ \\
\hline & & pomegranate & ------ & ----- & --.-- & $\mathrm{S} 1^{\mathrm{P} 3}$ & $\mathrm{~S} 2^{\mathrm{P} 3}$ & $\mathrm{~N}^{\mathrm{P} 3}$ & $\mathrm{~N}^{\mathrm{P} 3}$ & $\mathrm{~S} 3^{\mathrm{P} 3}$ \\
\hline & & Almond & ------ & ------ & ----- & $\mathrm{S} 2^{\mathrm{P} 3}$ & $\mathrm{~S} 2^{\mathrm{P} 3}$ & $\mathrm{~N}^{\mathrm{P} 3}$ & $\mathrm{~N}^{\mathrm{P} 3}$ & $\mathrm{~S} 3^{\mathrm{P} 3}$ \\
\hline
\end{tabular}

Abbreviations: S1: high suitable S2: Moderate suitable S3: Marginal suitable P1: high priority P2: moderate priority P3: less priority 
possible economically to construct water harvesting models especially earth, stone, or cement dyke comparing with the wide area of the table land and some open areas of the piedmont and coastal plain which are suitable for rainfed forge crops (barley) and in some cases constructing forage farms of Atriplex halimus, Sesbania sp., Leucaena leucocephala, Acacia sp., and Medicago arboria which could be planting using contouring models.

\section{Soil suitability and landform priority}

The circumstance the northern western coastal zone as well as the study area depends on rainwater harvesting in the streambed. Coarse-texture and moderately coarse-texture dominant in the study area and somehow the medium-texture soils are favorable for water harvesting. Whereas these types of soil textures have macropores and mesopores that receive the rainwater and makes it free and not subjected to evaporation. In addition to the traditional practices done by the local farmer including plowing the soil surface perpendicular on water flow direction line that enriches the stored water and prevent its upward movement.

For determining soil suitability of the chosen crops, namely; watermelon, olive, fig, wheat, barley, pomegranate, and almond, the soil mapping units were defined based on the variation surface slope, soil depth, and soil texture for each landform as shown in Table (3 and 4). Furthermore, the soil suitability for a soil mapping unit within each landform was assessed by comparing the weighted average of the measured soil characteristics with crop requirements to identify the soil suitability classes using LUSET program. Results indicated that all soil mapping units detecting in the coastal plain are marginal suitable (S3) for all of the selected crops due the higher soil salinity and alkalinity. Soil mapping unit 03 and 04 found in the coalesced deltas of the piedmont plain present high suitability for barley, olive, fig, pomegranate, and almond while they present a moderate suitability for watermelon and wheat.

On the other hand, the study revealed that SMU03 and 04 recognized in the drainage channel of both of piedmont plain and tableland in addition to SMU04 of tableland summit attained high suitable values for all studied crops except for watermelon which is moderate suitable. SMU05 of tableland summit is moderate suitable for watermelon, high suitable for barley and wheat, and marginal for orchard trees. The data shown in Table (3) revealed that SMU05 of the summit of the piedmont plain is moderate suitable for watermelon, barley, and wheat while it marginal suitable for orchard trees. On the other hand, SMU06 and 07 of the same landform are marginal suitable for watermelon, barley, and wheat and not suitable for all orchard trees. As for the tableland, soil mapping units of the drainage channel attained high suitable class for orchard trees and as well barley, while they were moderate suitable for watermelon and wheat. SMU05 of the summit of piedmont and tableland in addition to SMU 08 of the summit of tableland are moderate suitable for watermelon, barley, and wheat and marginal suitable for orchard trees. SMU06 and 07 are marginal suitable for watermelon, barley, and wheat and not suitable for orchard trees.

Three preferable classes were used to link landforms to best fitted crops to their best, namely; high priority (P1), moderate priority (P2), and less priority (P3), Table (4). In this sense, the coastal plain was high priority (P1) for Barley, moderate priority (P2) for wheat, and less priority (P3) for orchard tress and watermelon. These may due to the high salinity found in the coastal plain. The crops with class (P3) could be moved up to class (P2) in other areas of the coastal plain if the salinity is low. The coalesced deltas subunit of the piedmont plain is high priority (P1) for barley and wheat and moderate priority (P2) for the rest of the selected crops. The drainage channel of the piedmont plain and the tableland is high priority (P1) for orchard trees and watermelon and present a less priority class (P3) for barley and wheat. On contrary, the summit subunit of the piedmont plain and the tableland is high priority ( $\mathrm{P} 1)$ for barley and wheat, moderate priority (P2) for watermelon, and less priority (P3) for orchard tress.

\section{CONCLUSION}

The integration between the well known land evaluation system as Land Use Suitability Evaluation Tool (LUSET), which stands on the standards of FAO, and the local farmer's knowledge and expertise present a valuable information for land use planning especially for areas with its own circumstances as north western coast of Egypt. This region of Egypt depends mainly on rainfall for agricultural use, so it is called as rainfed region. The area under study, wadi sakher, is a case study represents a model for rainfed agricultural system. The current study focused on characterizing the land suitability by applying an international land suitability system taking in consideration the local community habit in agricultural use planning. It is found that the landforms and their elements which is covering the study play a vital role for crop selection. With respect to their own properties that affect the agricultural landuse whereas the drainage channel is suitable to be cultivated by orchard trees and rainfed watermelon. On the other 
hand, the summit of tableland and piedmont plain are preferred to be cultivated by cereal crops. Coalesced deltas has first priority for cereal crops and second priority for orchards and watermelon. Finally, the coastal plain has high priority for barley in contrast with other crops.

\section{REFERENCES}

AbdelRahman, M. A., A.Natarajan and R. Hegde. 2016. Assessment of land suitability and capability by integrating remote sensing and GIS for agriculture in Chamarajanagar district, Karnataka, India. The Egyptian Journal of Remote Sensing and Space Science 19:125141.

Aredehey, G., A.Mezgebu and A. Girma. 2018. Land-use land-cover classification analysis of Giba catchment using hyper temporal MODIS NDVI satellite images. International Journal of Remote Sensing 39:810-821.

Beek, K. J., K.De Bie and P. Driessen. 1997. Land information and land evaluation for land use planning and sustainable land management. The Land 1:27-44.

Doolette, J. 1986. The Diversity of Technology Needs in Rainfed agriculture: the Case Study of North Africa. In: Davis, T. 1. (ed.). Developments of Rainfed Agriculture Under Arid and Semiarid Conditions. Proceedings of the Sixth Agriculture Sector Symposium held at the World Bank 6-10 January 1986. Washington D.C. USA. pp 416.

El Shazly, E., M. Abdel-Hady, M.El Ghawaby , I. El Kassas, S. Khawasik, M.El Shazly and S.Sanad. 1975. Geologic interpretation of Landsat satellite images for west Nile delta area, Egypt. Cairo, Egypt: The Remote Sensing Research Project, Academy of Scientific Research \& Technology.

FAO 1976. "A frame work for land evaluation. Soil Bulletin 32, Soil resources development and conservation services, Land and Water Development Division (AGLS), FAO HQ. Rome.." ILRI.

FAO 2007. "'Land evaluation: Towards a revised framework" Land \& Water Discussion Paper 6; Rome."

Hansen, M. C., R. S. DeFries, J. R. Townshend and R.Sohlberg. 2000. Global land cover classification at $1 \mathrm{~km}$ spatial resolution using a classification tree approach. International journal of remote sensing 21:1331-1364.

Heathcote, R. L. 1983. "The Arid Lands: Their use and abuse. Longman, New York, USA," Longman.

Jahn, R., H. Blume, V.Asio, O.Spaargaren, and P.Schad. 2006. "Guidelines for soil description," FAO.

Kamphorst, A., and G. Bolt. 1976. Saline and sodic soils. In "Developments in Soil Science". 5. pp:171-191. Elsevier.

Lal, R. 1994 Sustainable land use systems and soil resilience. In Soil Resilience and Sustainable land use (ed. D.J. Greenland \& I. Szabolcs), Wallingford, UK: CAB International: 41-67.
Liang, S. 2008. "Advances in land remote sensing: system, modeling, inversion and application," Springer Science \& Business Media.

Liu, Y., and Y.Chen. 2006. Impact of population growth and land-use change on water resources and ecosystems of the arid Tarim River Basin in Western China. The International Journal of Sustainable Development \& World Ecology. 13: 295-305.

Merolla, S., G.Armesto, and G.Calvanese.1994. A GIS application for assessing agricultural land. ITC Journal: 264-269.

Rao, D., N.Gautam, R.Nagaraja, and P. R.Mohan. 1996. IRS$1 \mathrm{C}$ applications in land use mapping and planning. Current Science: 575-581.

Rashidi, M., and M. Seilsepour. 2008. Modeling of soil exchangeable sodium percentage based on soil sodium adsorption ratio. International Journal of Agriculture \& Biology 3:22-6.

Samaniego, L., and K.Schulz. 2009. Supervised classification of agricultural land cover using a modified k-NN technique $(\mathrm{MNN})$ and Landsat remote sensing imagery. Remote Sensing 1: 875-895.

Sathish, A., and K.Niranjana. 2010. Land suitability studies for major crops in Pavagada taluk, Karnataka using remote sensing and GIS techniques. Journal of the Indian Society of Remote Sensing 38:143-151.

Schoeneberger, P. J. 2012. "Field book for describing and sampling soils," Government Printing Office.

Soil Science Division Staff . 2017. "Soil survey manual. C. Ditzler, K. Scheffe, and H.C. Monger (eds.). USDA Handbook 18. Government Printing Office, Washington, D.C.."

Soil Survey Staff. 2014. "Keys to soil taxonomy," Department of Agriculture: Natural Resources Conservation Service.

Sys, I. C., E.Van Ranst, I. J.Debaveye and F. Beenaert. 1993. Land evaluation (Part I-III). Crop Requirements. 199p. General Administration for Development Cooperation, Brussels. Belgium.

USDA .2004. Soil survey laboratory methods manual. Soil survey investigations report 42.

Yadav, P., M. Kapoor and K.Sarma. 2012. Land use land cover mapping, change detection and conflict analysis of Nagzira-Navegaon Corridor, Central India using geospatial technology. International Journal of Remote Sensing and GIS 1: 90-98.

Yan, E., G.Wang, H. Lin, C. Xia, and H. Sun. 2015. Phenology-based classification of vegetation cover types in Northeast China using MODIS NDVI and EVI time series. International Journal of Remote Sensing 36: 489512.

Yen, B. T., K. S.Pheng. and C. T. Hoanh. 2006. Land use suitability evaluation tool user's guide. INTERNATIONAL RICE RESEARCH INSTITUTE DAPO Box 7777 Metro Manila Philippines., 15. 


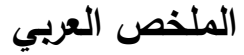

\section{تحديد صلاحية الأراضي لانتاج المحاصيل و الفاكهة بوادي صخر بالساحل الشمالي الغزبي لمصر}

$$
\text { عبدالصمد عبدالستار علي الضبع }
$$

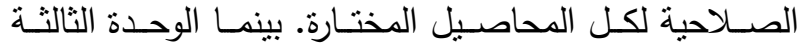
والرابعـة والتـي تم تحديدها في كل مـن الدلتاوات الملتحمـة وقنوات الصرف للسـهل البيدمنونتي بالاضـافة إلي الوحدة الرابعة في القمة للهضبة ذات صلاحية عالية لكل المحاصيل المختارة عدا البطيخ حيث أظهر صـلاحية متوسطة. بينما الوحدة الخامسـة والتـي تحققت في القــة لكل مـن الســل البيـدمونتي والهضـبة وكـذلك الوحـدة الثامنـة لقــة الهضـبة أظهرت صـلاحية متوسطة لكل من البطيخ والثـعير والقهح بينمـا كانت هامشية الصـلاحية للمحاصبيل البستانية. أخيـر

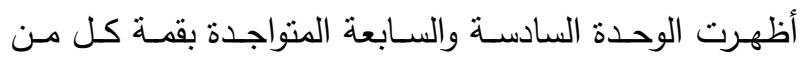
السهل البيدمونتي والهضبة أنهما هامشي الصـلاحية لكل من

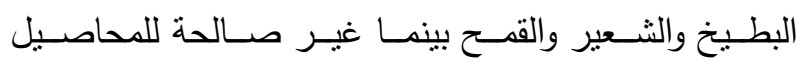
البستانية المختارة. بالنسبة لأولوية الأشكال الارضية للزراعة ولئرة

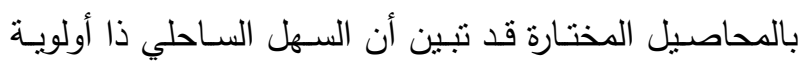

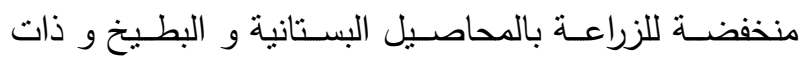
أولوية عالية ومتوسطة لكل من الثعبر والقمح علي التوالي.

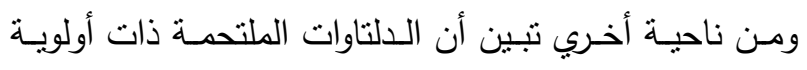
عالية لكل من الثعير والقـح ومتوسطة لكل من البطيخ و

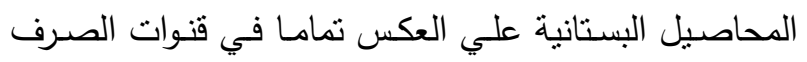
التـي ابـدت أولويسة مرتفعـة لكـل مـن المحاصـيل البسـتانية

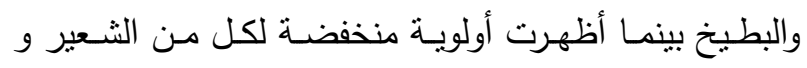
القمـح. بالنسـبة لقمـة كل مـن الســل البيـمونتي و الهضـبة

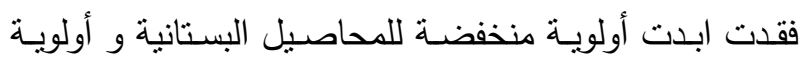
متوسطة للبطيخ و أولوية مرتفعة للشعير و القمح.
تعبـر صـلاحية الارض علـي مـدي ملائمسة المحاصسيل

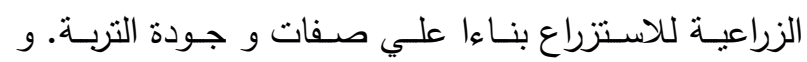

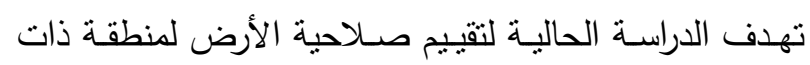
خصسائص خاصسة حيـث تعتمـد علي حصساد ميـاه الامطسار الموسمية في عملية الزراعة. في هذا الصدد تم عمل تكامل بين أحد نظم تقييم صلاحية الأراضي مع الاعتماد علي خبرة

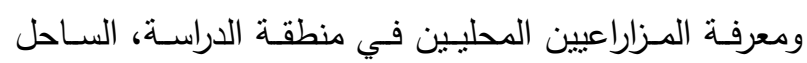
الثـمالي الغربـي لمصـر عامـة. بنــاءا علـي معرفـة وخبـرة المزارعين فإن الدراسة الحالية ركزت علي الاشكال الارضية

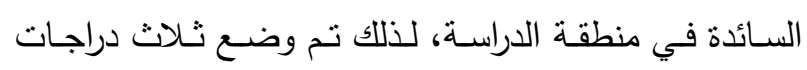
تصنيفية حيث تعبر عن مدي أولوية شكل الارض للمحاصيل

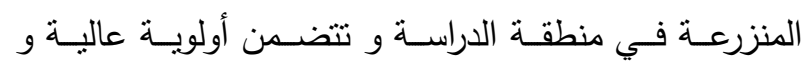

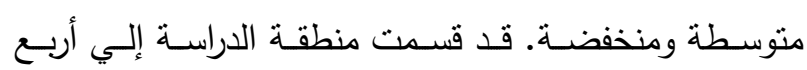
وحدات من الاثكال الارضية و هي السهل السـاحلي، السهل البيدمونتي، والمنحدر ، و الهضبة. وقد تم اعادة تقسيم السهل

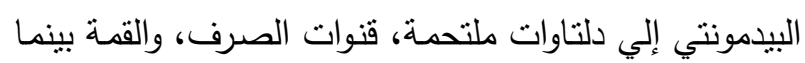

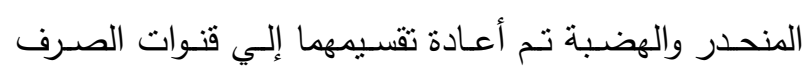

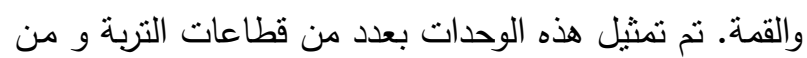

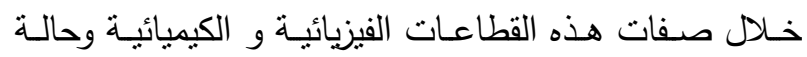
الخصوبة تم استتباط م وحدات خرائطية للتربة. و قد بينت

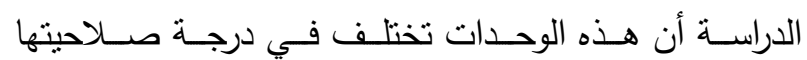
للمحاصيل المختارةو هي البطيخ، الثعير ، القمح، والزيتون،

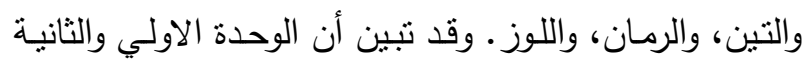

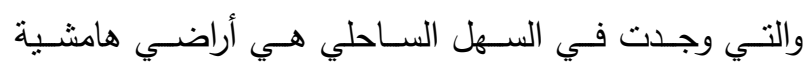

